\title{
A Parametrised Functional Interpretation of Heyting Arithmetic
}

\author{
Bruno Dinis $^{\mathrm{a}, 1}$, Paulo Oliva ${ }^{\mathrm{b}}$ \\ ${ }^{a}$ Departamento de Matemática, Faculdade de Ciências da Universidade de Lisboa, Campo \\ Grande, Ed. C6, 1749-016, Lisboa, Portugal \\ ${ }^{b}$ School of Electronic Engineering and Computer Science, Queen Mary University of London, \\ London E1 4NS, United Kingdom
}

\begin{abstract}
This paper presents an abstract parametrised functional interpretation of WE-HA ${ }^{\omega}$. It is based on families of parameters allowing for different degrees of freedom on the design of concrete interpretations. In this way, we are able to generalise previous work on unifying functional interpretations by including in the unification the more recent bounded and Herbrandized functional interpretations.

Keywords: functional interpretations, unification, intuitionism, proof theory 2000 MSC: 03F07, 03F10, 03F30, 03F55
\end{abstract}

\section{Introduction}

Since Gödel [16] published his functional ("Dialectica") interpretation in 1958, various other functional interpretations have been proposed ${ }^{2}$. These include Kreisel's modified realizability [20], the Diller-Nahm variant of the Dialectica interpretation [4], Stein's family of interpretations [28], and more recently, the bounded functional interpretation [12], the bounded modified realizability [11], and "Herbrandized" versions of modified realizability and the Dialectica [3]. In view of this picture, several natural questions arise: How are these different interpretations related to each other? What is the common structure behind all of them?

Email addresses: bmdinis@fc.ul.pt (Bruno Dinis), p.oliva@qmul.ac.uk (Paulo Oliva)

${ }^{1}$ The author acknowledges the support of FCT - Fundação para a Ciência e Tecnologia under the projects UIDB/04561/2020 and UIDP/04561/2020, and the research center Centro de Matemática, Aplicações Fundamentais e Investigação Operacional, Universidade de Lisboa.

${ }^{2}$ See [2] for a survey on the "Dialectica" interpretation. 
Are there any other interpretations out there waiting to be discovered?

These questions were addressed by the second author and various co-authors in a series of papers on unifying functional interpretations. Starting with a unification of interpretations of intuitionistic logic [21], which was followed by various analysis of functional interpretations within the finer setting of linear logic $[13,22,23,24]$, a proposal on how functional interpretations could actually be combined in the so-called hybrid functional interpretations [17, 25], and the inclusion of truth variants in the unification [15].

Functional interpretations associate with each formula $A$ a new formula $\left\{\{A\}_{y}^{x}\right.$ where $\boldsymbol{x}$ and $\boldsymbol{y}$ are fresh tuples of variables. Intuitively, $\boldsymbol{x}$ captures the "positive" quantifications in $A$, while $\boldsymbol{y}$ captures the "negative" quantifications. This is done in such a way that, in a suitable system, the truth of $A$ is equivalent to that of $\exists \boldsymbol{x} \forall \boldsymbol{y}\{\{A\}\}_{\boldsymbol{y}}^{\boldsymbol{x}}$. The key insight which arises from the programme of "unifying functional interpretations" is that we have some degree of freedom when choosing the interpretation of the exponentials of linear logic!A and ?A. For instance, we can take

$$
\begin{array}{ll}
\{! A\}\}_{y}^{x}: \equiv !\{\{A\}\}_{y}^{x} & \text { (giving rise to the Dialectica interpretation) } \\
\left\{\{! A\}_{a}^{x}: \equiv ! \forall y \in a\left\{\{A\}_{y}^{x}\right.\right. & \text { (giving rise to the Diller-Nahm interpretation) } \\
\{\{! A\}\}^{x}: \equiv ! \forall y\left\{\{A\}_{y}^{x}\right. & \text { (giving rise to modified realizability) } \\
\{! ! A\}\}^{x}: \equiv ! \forall y\left\{\{A\}_{y}^{x} \otimes ! A\right. & \text { (giving rise to modified realizability with truth) } \\
\text { and so on... } &
\end{array}
$$

showing that these interpretations only differ in the way they treat the contraction axiom. In particular, in the pure fragment of linear logic all these interpretations coincide!

So, it makes sense to introduce an abstract bounded quantification $\forall x \sqsubset_{\tau} a A$, capturing this degree of freedom on the design of a functional interpretation, and to try to isolate the properties of this parameter which ensure the soundness of the interpretation. With this, one is able to define a "unifying functional interpretation" which when instantiated gave rise to several of the existing functional interpretations, including the Dialectica interpretation, modified realizability (its q- and truth variants), Stein's family of interpretations, and the Diller-Nahm interpretation [21, 24]. This process also led to the design of a "Diller-Nahm with truth" interpretation [15], which at the time was thought not to be possible.

However, the unifying functional interpretation programme has so far been unable to capture the two more recent families of functional interpretations, namely 
the bounded functional interpretations $[6,10,11,12]$, and the Herbrandized functional interpretations [3, 9].

In this paper we propose a more general parametrisation of the functional interpretation, introducing other families of parameters which allows us to capture different interpretations of typed quantifications. We demonstrate that, when devising a functional interpretation, we in fact have two crucial degrees of freedom: we can choose how to interpret the contraction axiom, as discussed above, but also, we can choose how to interpret typed quantifications, which ultimately boils down to the choice of how predicate symbols are interpreted.

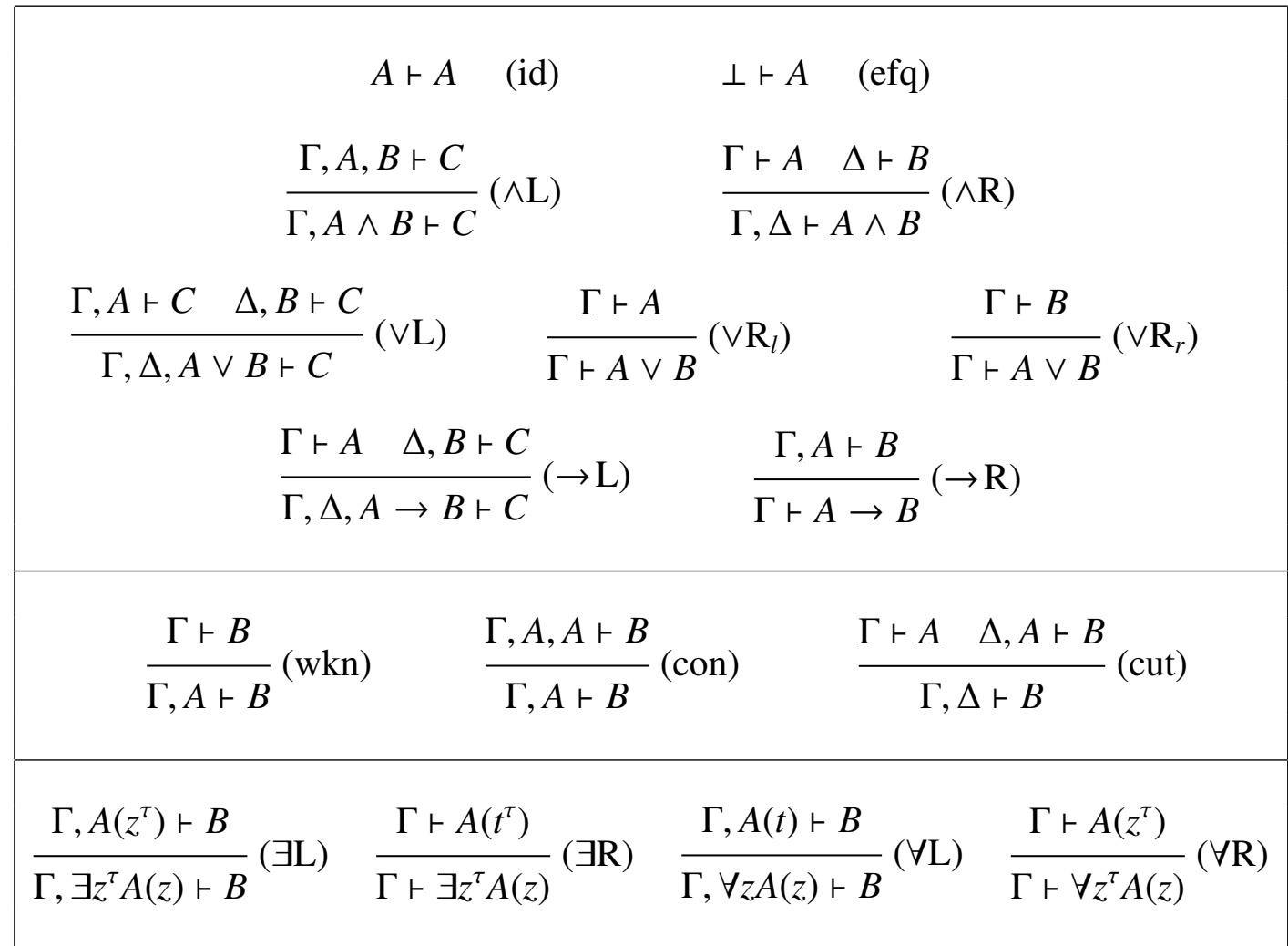

Table 1: Logical axioms and rules of WE-HA ${ }^{\omega}$

In this paper, we will carry out the details in the context of Heyting arithmetic in all finite types WE-HA ${ }^{\omega}$, but the same can also be done for other theories, with suitable assumptions for the interpretation of its non-logical axioms. In [7], we explain how this parametrised interpretation of Heyting arithmetic arises from 
a parametrised interpretation of intuitionistic linear logic combined with translations of intuitionistic logic into intuitionistic linear logic.

The parametrised interpretation of WE-HA ${ }^{\omega}$ will be presented in Section 2. In Section 3 we show how instantiating the different parameters then gives rise to each of the functional interpretations mentioned above. In this process we have again discovered some new functional interpretations.

\section{A Parametrised Functional Interpretation}

We will fix the "source" theory as WE-HA ${ }^{\omega}$, while the "target" theory $\mathcal{A}_{\mathbf{t}}$ might be a strict extension of WE-HA ${ }^{\omega}$. We will add only enough assumptions on $\mathcal{A}_{\mathbf{t}}$ to ensure that the soundness of WE-HA ${ }^{\omega}$ into $\mathcal{A}_{\mathbf{t}}$ goes through. Our assumptions are as follows:

(A1) The target theory $\mathcal{A}_{\mathbf{t}}$ is an extension of WE-HA ${ }^{\omega}$.

(A2) For each finite type ${ }^{3} \rho$ we have, in the target theory $\mathcal{A}_{\mathbf{t}}$, a formula $x<^{\rho} a$, and a finite type $\operatorname{wt}(\rho)$. We will call $\operatorname{wt}(\rho)$ the witnessing type of $\rho$. We write $\forall x \prec^{\rho} a A$ and $\exists x<^{\rho} a A$ as abbreviations for $\forall x^{\rho}\left(x<^{\rho} a \rightarrow A\right)$ and $\exists x^{\rho}\left(x<^{\rho} a \wedge A\right)$, respectively.

(A3) For each finite type $\rho$ we also have, in the target theory $\mathcal{A}_{\mathbf{t}}$, a formula $\mathrm{W}_{\rho}(x)$, which we will use to restrict the domain of the witnesses and counterwitnesses. We assume that $x<^{\rho} a$ implies that $a$ is in $\mathrm{W}_{\mathrm{wt}(\rho)}$, i.e.

$$
x<^{\rho} a \vdash_{\mathcal{A}_{\mathbf{t}}} \mathrm{W}_{\mathrm{wt}(\rho)}(a) .
$$

When $\tau$ is a tuple of finite types $\tau_{1}, \ldots, \tau_{n}$, we write $\mathrm{W}_{\tau}\left(x_{1}, \ldots, x_{n}\right)$ as an abbreviation for $\mathrm{W}_{\tau_{1}}\left(x_{1}\right), \ldots, \mathrm{W}_{\tau_{n}}\left(x_{n}\right)$, when it appears in the context of a sequent, or for $\mathrm{W}_{\tau_{1}}\left(x_{1}\right) \wedge \ldots \wedge \mathrm{W}_{\tau_{n}}\left(x_{n}\right)$, when it appears in the conclusion of a sequent. We assume that, provably in $\mathcal{A}_{\mathbf{t}}$, the combinators $\mathrm{S}_{\rho, \tau, \sigma}$ and $\mathrm{K}_{\rho, \tau}$ are in $\mathrm{W}$, and that the application of a function in $\mathrm{W}$ to an $\operatorname{argument}$ in $\mathrm{W}$ will also be in $\mathrm{W}$, i.e.

$$
\begin{aligned}
& \left(\mathbf{W}_{\mathrm{K}}\right) \vdash_{\mathcal{A}_{\mathbf{t}}} \mathrm{W}_{\rho \rightarrow \tau \rightarrow \rho}\left(\mathrm{K}_{\rho, \tau}\right) \\
& \left(\mathbf{W}_{\mathrm{S}}\right) \vdash_{\mathcal{A}_{\mathbf{t}}} \mathbf{W}_{(\rho \rightarrow \tau \rightarrow \sigma) \rightarrow(\rho \rightarrow \tau) \rightarrow \rho \rightarrow \sigma}\left(\mathbf{S}_{\rho, \tau, \sigma}\right) \\
& \left(\mathbf{W}_{\mathrm{Ap}}\right) \mathrm{W}_{\tau}(x), \mathbf{W}_{\tau \rightarrow \rho}(f) \vdash_{\mathcal{A}_{\mathbf{t}}} \mathbf{W}_{\rho}(f x)
\end{aligned}
$$

\footnotetext{
${ }^{3}$ For the sake of simplicity, we will take the booleans $\mathbb{B}$ as a primitive base finite type, although in WE-HA ${ }^{\omega}$ the booleans are normally defined from the natural numbers $\mathbb{N}$ by interpreting 0 as true, and $n>0$ as false. This means that for us, the atomic formulas of $\mathbf{W E}-\mathbf{H A} \mathbf{A}^{\omega}$ are $s={ }_{\mathbb{B}} t$ (for $s, t: \mathbb{B})$ and $s=\mathbb{N} t($ for $s, t: \mathbb{N})$.
} 
(A4) For each formula $A$ of $\mathcal{A}_{\mathbf{t}}$, tuple of variables $\boldsymbol{x}=x_{1}, \ldots, x_{n}$, and finite types $\tau=\tau_{1}, \ldots, \tau_{n}$ we associate a tuple of bounding types bt $(\tau)$ and a formula $\forall \boldsymbol{x} \sqsubset_{\tau} \boldsymbol{a} A$, in which the variables $\boldsymbol{x}$ are no longer free. If $\Gamma=A_{1}, \ldots, A_{n}$ then $\forall \boldsymbol{x} \sqsubset_{\tau} \boldsymbol{a} \Gamma$ abbreviates $\forall \boldsymbol{x} \sqsubset_{\tau} A_{1}, \ldots, \forall \boldsymbol{x} \sqsubset_{\tau} A_{n}$. We do not assume that the tuple of finite types $\operatorname{bt}(\tau)$ has the same length as $\tau$. The intuition is that $\boldsymbol{x}$ ranges over elements of type $\boldsymbol{\tau}$, whereas the bounds $\boldsymbol{a}$ range over possibly different types bt $(\tau)$. The formula construction $\forall \boldsymbol{x} \sqsubset_{\tau} \boldsymbol{a} A$ is assumed to satisfy the following properties (so that it indeed behaves as a bounded universal quantifier):

$\left(\mathbf{Q}_{1}\right)$ If $\Gamma \vdash_{\mathcal{A}_{\mathrm{t}}} A$ then $\forall \boldsymbol{x} \sqsubset_{\tau} a \Gamma \vdash_{\mathcal{A}_{\mathrm{t}}} \forall \boldsymbol{x} \sqsubset_{\tau} a A$

$\left(\mathbf{Q}_{2}\right) \forall \boldsymbol{x}^{\mathrm{W}} A \vdash_{\mathcal{A}_{\mathrm{t}}} \forall \boldsymbol{x} \sqsubset_{\tau} \boldsymbol{a} A$ (and in particular $\forall \boldsymbol{x} \sqsubset_{\tau} \boldsymbol{a} \mathrm{W}_{\tau}(\boldsymbol{x})$ )

$\left(\mathbf{Q}_{3}\right) \forall \boldsymbol{x} \sqsubset_{\tau} \boldsymbol{a} A \vdash_{\mathcal{A}_{\mathbf{t}}} A$, if $\boldsymbol{x}$ is not free in $A$

$\left(\mathbf{Q}_{4}\right) \forall \boldsymbol{x} \sqsubset_{\tau} \boldsymbol{a}(A(x) \wedge B) \vdash_{\mathcal{A}_{\mathbf{t}}} \forall \boldsymbol{x} \sqsubset_{\tau} \boldsymbol{a} A(x) \wedge B$, if $\boldsymbol{x}$ is not free ${ }^{4}$ in $B$.

For each formula $A$ of $\mathcal{A}_{\mathbf{t}}$, tuple of variables $\boldsymbol{x}$, and types $\boldsymbol{\tau}$ and $\rho$ we also assume that there exist terms $\boldsymbol{\eta}(\cdot),(\cdot) \sqcup(\cdot)$ and $(\cdot) \circ(\cdot)$ of $\mathcal{A}_{\mathbf{t}}$ such that

$\left(\mathbf{C}_{\eta}\right) \mathrm{W}_{\tau}(z), \forall \boldsymbol{x} \sqsubset_{\tau} \boldsymbol{\eta}(z) A \vdash_{\mathcal{A}_{\mathbf{t}}} A[z / \boldsymbol{x}]$

$\vdash_{\mathcal{A}_{\mathrm{t}}} \mathrm{W}_{\tau \rightarrow \mathrm{bt}(\tau)}(\boldsymbol{\eta})$

$\left(\mathbf{C}_{\sqcup}\right) \mathrm{W}_{\tau, \tau}\left(x_{1}, x_{2}\right), \forall x \sqsubset_{\tau}\left(x_{1} \sqcup x_{2}\right) A \vdash_{\mathcal{A}_{t}} \forall x \sqsubset_{\tau} x_{1} A \wedge \forall x \sqsubset_{\tau} x_{2} A$

$\vdash_{\mathcal{A}_{\mathrm{t}}} \mathrm{W}_{\mathrm{bt}(\tau) \rightarrow \mathrm{bt}(\tau) \rightarrow \mathrm{bt}(\tau)}\left(\lambda \boldsymbol{x}_{1}, \boldsymbol{x}_{2} . \boldsymbol{x}_{1} \sqcup \boldsymbol{x}_{2}\right)$

$\left(\mathbf{C}_{\circ}\right) \mathrm{W}_{\rho \rightarrow \mathrm{bt}(\tau)}(f), \mathrm{W}_{\mathrm{bt}(\rho)}(z), \forall \boldsymbol{x} \sqsubset_{\tau}(f \circ z) A \vdash_{\mathcal{A}_{\mathrm{t}}} \forall \boldsymbol{y} \sqsubset_{\rho} z \forall \boldsymbol{x} \sqsubset_{\tau} f \boldsymbol{y} A$

$\vdash \mathcal{A}_{\mathrm{t}} \mathrm{W}_{(\rho \rightarrow \mathrm{bt}(\tau)) \rightarrow \mathrm{bt}(\rho) \rightarrow \mathrm{bt}(\tau)}(\lambda \boldsymbol{f}, \boldsymbol{z} . \boldsymbol{f} \circ \boldsymbol{z})$

(A5) Assume that for each finite type $\rho$ of $\mathbf{W E - H A}{ }^{\omega}$ we have a term in $\mathcal{A}_{\mathbf{t}}$

$$
\mathrm{m}_{\rho}^{\mathbb{B}}: \operatorname{wt}(\mathbb{B}) \rightarrow \operatorname{wt}(\rho) \rightarrow \operatorname{wt}(\rho) \rightarrow \operatorname{wt}(\rho)
$$

such that ${ }^{5}$

(i) $\vdash_{\mathcal{A}_{\mathrm{t}}} \mathrm{W}_{\mathrm{wt}(\mathbb{B}) \rightarrow \mathrm{wt}(\rho) \rightarrow \mathrm{wt}(\rho) \rightarrow \mathrm{wt}(\rho)}\left(\mathrm{m}_{\rho}^{\mathbb{B}}\right)$, and

(ii) $\mathrm{W}\left(a_{1}, a_{2}\right), z \prec^{\mathbb{B}} b, \boldsymbol{x} \prec^{\rho} \operatorname{if}_{\rho}\left(z, a_{1}, a_{2}\right) \vdash_{\mathcal{A}_{\mathbf{t}}} \boldsymbol{x} \prec^{\rho} \mathrm{m}_{\rho}^{\mathbb{B}}\left(b, a_{1}, a_{2}\right)$.

See Definition 2.6 and Lemma 2.7 where the operation $\mathrm{m}_{\rho}^{\mathbb{B}}$ is lifted to arbitrary formulas $A$.

(A6) Assume that for each finite type $\rho$ of $\mathbf{W E - H A}{ }^{\omega}$ we have a term in $\mathcal{A}_{\mathbf{t}}$

$$
\mathrm{m}_{\rho}^{\mathbb{N}}:(\mathbb{N} \rightarrow \operatorname{wt}(\rho)) \rightarrow \operatorname{wt}(\mathbb{N}) \rightarrow \operatorname{wt}(\rho)
$$

such that

\footnotetext{
${ }^{4}$ It is easy to check that $\left(\mathbf{Q}_{4}\right)$ in fact follows from $\left(\mathbf{Q}_{1}\right)$ and $\left(\mathbf{Q}_{3}\right)$, but we include it here as an assumption for future reference.

${ }^{5}$ We are denoting by "if ${ }_{\rho}\left(z^{\mathbb{B}}, x_{1}^{\rho}, x_{2}^{\rho}\right)$ " the family of terms definable in $\mathbf{W E}-\mathbf{H A}{ }^{\omega}$ such that if $_{\rho}\left(\mathrm{T}, x_{1}, x_{2}\right)={ }_{\rho} x_{1}$ and if $\rho$ (F, $\left.x_{1}, x_{2}\right)={ }_{\rho} x_{2}$, though we will often omit the type in the subscript.
} 
- $\vdash_{\mathcal{A}_{\mathbf{t}}} \mathrm{W}_{(\mathbb{N} \rightarrow \mathrm{wt}(\rho)) \rightarrow \mathrm{wt}(\mathbb{N}) \rightarrow \operatorname{wt}(\rho)}\left(\mathrm{m}_{\rho}^{\mathbb{N}}\right)$

- $\forall n^{\mathbb{N}} \mathrm{W}(f n), n<^{\mathbb{N}} a, \boldsymbol{x}<^{\rho} f n \vdash_{\mathcal{A}_{\mathbf{t}}} \boldsymbol{x}<^{\rho} \mathrm{m}_{\rho}^{\mathbb{N}}(f)(a)$

See Definition 2.8 and Lemma 2.9 where the operation $\mathrm{m}_{\rho}^{\mathbb{N}}$ is lifted to arbitrary formulas $A$. We also assume we have a terms $N^{\mathrm{wt}(\mathbb{N}) \rightarrow \mathbb{N}}$ and $v^{\mathbb{N} \rightarrow \operatorname{wt}(\mathbb{N})}$ such that $n \prec^{\mathbb{N}} a \vdash_{\mathcal{A}_{\mathbf{t}}} n \leq N a$ and $\vdash_{\mathcal{A}_{\mathbf{t}}} n \prec^{\mathbb{N}} v n$ (and hence $n \leq N(v n)$ ).

(A7) Assume that for each term $\left(t\left[\boldsymbol{x}^{\sigma}\right]\right)^{\tau}$ of $\mathbf{W E}-\mathbf{H A}^{\omega}$ we have a term $\left(\tilde{t}\left[\boldsymbol{a}^{\mathrm{wt}(\boldsymbol{\sigma})}\right]\right)^{\mathrm{wt}(\tau)}$ in $\mathcal{A}_{\mathbf{t}}$ such that

$$
\text { - } \boldsymbol{x} \prec^{\sigma} \boldsymbol{a} \vdash_{\mathcal{A}_{\mathrm{t}}} t[\boldsymbol{x}]<^{\tau} \tilde{t}[\boldsymbol{a}]
$$

In particular, it follows that for each boolean constant $b^{\mathbb{B}}$ there exists a term $\tilde{b}^{\text {wt(B) }}$ such that $\vdash_{\mathcal{A}_{\mathrm{t}}} b<^{\mathbb{B}} \tilde{b}$, and for each numeral $n^{\mathbb{N}}$ there exists a term $\tilde{n}^{\text {wt( }(\mathbb{N})}$ such that $\vdash_{\mathcal{A}_{\mathbf{t}}} n \prec^{\mathbb{N}} \tilde{n}$.

Lemma 2.1. Let $t[\boldsymbol{a}]$ be a term of $\mathcal{A}_{\mathbf{t}}$ built from variables $\boldsymbol{a}=a_{1}, \ldots, a_{n}$ and the combinators $\mathrm{K}$ and $\mathrm{S}$ via application. Then $\mathrm{W}\left(a_{1}, \ldots, a_{n}\right) \vdash_{\mathcal{A}_{\mathbf{t}}} \mathrm{W}(t[\boldsymbol{a}])$.

Proof. By induction on the structure of $t$, using $\left(\mathbf{W}_{\mathrm{S}}\right),\left(\mathbf{W}_{\mathrm{K}}\right)$, and $\left(\mathbf{W}_{\mathrm{Ap}}\right)$.

In each concrete instantiation of the parametrised interpretation we will consider different choices for the parameters $\left\{x \prec^{\rho} a\right\}_{\rho \in \mathcal{T}},\{\mathrm{Wt}(\rho)\}_{\rho \in \mathcal{T}},\left\{\mathrm{W}_{\tau}(x)\right\}_{\tau \in \mathcal{T}}$, and $\left\{\forall \boldsymbol{x} \sqsubset_{\tau} \boldsymbol{a} A\right\}_{A \in \mathbf{F o r m}_{\mathcal{H}_{\mathbf{t}}}, \tau \in \mathcal{T}}$.

Definition 2.2 (Adequate parameters). A choice of parameters will be called adequate for $\mathcal{A}_{\mathbf{t}}$ if assumptions (A1) - (A7) hold. Given a class of formulas $C \subseteq$ Form $_{\mathcal{A}_{\mathbf{t}}}$, we say that the choice of parameters in $\mathcal{A}_{\mathbf{t}}$ is $C$-adequate for $\mathcal{A}_{\mathbf{t}}$ if it is adequate for $\mathcal{A}_{\mathbf{t}}$ when assumption (A4) is only required to hold for formulas in $C$.

Notation 2.3. Given tuples of types $\rho=\rho_{1}, \ldots, \rho_{n}$ and $\tau=\tau_{1}, \ldots, \tau_{m}$ we write $\rho \rightarrow \tau$ as an abbreviation for the tuple of types

$$
\rho_{1} \rightarrow \ldots \rightarrow \rho_{n} \rightarrow \tau_{1}, \ldots, \rho_{1} \rightarrow \ldots \rightarrow \rho_{n} \rightarrow \tau_{m}
$$

Given tuples of terms $\boldsymbol{t}=t_{1}, \ldots, t_{n}$ and $\boldsymbol{s}$ we write $\boldsymbol{t}$ s for the tuple $t_{1} \boldsymbol{s}, \ldots, t_{n} \boldsymbol{s}$.

\subsection{Witnessing types}

Let us write $\varepsilon$ for the empty tuple of terms or the empty tuple of types. 
Definition 2.4. We generalise the notion of witnessing type to all formulas by defining for each formula A tuples of types $\tau_{A}^{+}$and $\tau_{A}^{-}$inductively as follows:

$$
\begin{array}{llll}
\tau_{P}^{+} & : \equiv \varepsilon, \quad \text { for P atomic } & \tau_{P}^{-} & : \equiv \varepsilon, \text { for P atomic } \\
\tau_{A \wedge B}^{+}: \equiv \tau_{A}^{+}, \tau_{B}^{+} & \tau_{A \wedge B}^{-}: \equiv \tau_{A}^{-}, \tau_{B}^{-} \\
\tau_{A \vee B}^{+}: \equiv \operatorname{wt}(\mathbb{B}), \tau_{A}^{+}, \tau_{B}^{+} & \tau_{A \vee B}^{-}: \equiv \operatorname{bt}\left(\tau_{A}^{-}\right), \operatorname{bt}\left(\tau_{B}^{-}\right) \\
\tau_{A \rightarrow B}^{+}: \equiv \tau_{A}^{+} \rightarrow \tau_{B}^{+}, \tau_{A}^{+} \rightarrow \tau_{B}^{-} \rightarrow \operatorname{bt}\left(\tau_{A}^{-}\right) & \tau_{A \rightarrow B}^{-}: \equiv \tau_{A}^{+}, \tau_{B}^{-} \\
\tau_{\exists z^{\rho} A}^{+}: \equiv \operatorname{wt}(\rho), \tau_{A}^{+} & \tau_{\exists z^{\rho} A}^{-}: \equiv \operatorname{bt}\left(\tau_{A}^{-}\right) \\
\tau_{\forall z^{\rho} A}^{+}: \equiv \operatorname{wt}(\rho) \rightarrow \tau_{A}^{+} & \tau_{\forall z^{\rho} A}^{-}: \equiv \operatorname{wt}(\rho), \tau_{A}^{-}
\end{array}
$$

Given a tuple of formulas $\Gamma=A_{1}, \ldots, A_{n}$ we write $\tau_{\Gamma}^{+}$(resp., $\tau_{\Gamma}^{-}$) for the tuple $\tau_{A_{1}}^{+}, \ldots, \tau_{A_{n}}^{+}\left(\operatorname{resp} . \tau_{A_{1}}^{-}, \ldots, \tau_{A_{n}}^{-}\right)$.

\subsection{Parametrised interpretation}

We can now present the parametrised interpretation of $\mathbf{W E - H A}{ }^{\omega}$ into $\mathcal{A}_{\mathbf{t}}$ :

Definition 2.5 (Parametrised interpretation). For each formula A of WE-HA ${ }^{\omega}$, let us associate a formula $\left\{\{A\}_{y}^{x}\right.$ of $\mathcal{A}_{\mathbf{t}}$, with two fresh tuples of free-variables $\boldsymbol{x}$ and $\boldsymbol{y}$, inductively as follows:

$$
\begin{aligned}
& \left\{\left\{s={ }_{\tau} t\right\}\right\}_{\varepsilon}^{\varepsilon} \quad \equiv s=t, \quad \tau \in\{\mathbb{B}, \mathbb{N}\} \\
& \left\{\{ A \wedge B \} _ { y , w } ^ { x , v } \equiv \left\{\{A\}_{y}^{x} \wedge\{\{B\}\}_{w}^{v}\right.\right. \\
& \left\{\{A \vee B\}_{y_{\boldsymbol{y}, \boldsymbol{w}}^{b, \boldsymbol{v}, \boldsymbol{v}}} \equiv \exists z<^{\mathbb{B}} b\left(\left(z=\mathrm{T} \wedge \forall \boldsymbol{y}^{\prime} \sqsubset_{\boldsymbol{\tau}_{A}^{-}} \boldsymbol{y}\left\{\{A\}_{\boldsymbol{y}^{\prime}}^{\boldsymbol{x}}\right) \vee\left(z=\mathrm{F} \wedge \forall \boldsymbol{w}^{\prime} \sqsubset_{\boldsymbol{\tau}_{B}^{-}} \boldsymbol{w}\left\{\{B\}_{\boldsymbol{w}^{\prime}}^{\boldsymbol{v}}\right)\right)\right.\right.\right. \\
& \left\{\{ A \rightarrow B \} _ { x , w } ^ { f , g } \equiv \forall \boldsymbol { y } \sqsubset _ { \tau _ { A } ^ { - } } g x w \left\{\{A\}_{y}^{x} \rightarrow\{\{B\}\}_{w}^{f x}\right.\right. \\
& \left\{\left\{\exists z^{\tau} A\right\}_{y}^{c, x} \equiv \exists z<^{\tau} c \forall \boldsymbol{y}^{\prime} \sqsubset_{\tau_{A}^{-}} \boldsymbol{y}\{\{A\}\}_{y^{\prime}}^{\boldsymbol{x}}\right. \\
& \left\{\left\{\forall z^{\tau} A\right\}_{c, \boldsymbol{y}}^{f} \equiv \forall z<^{\tau} c\left\{\{A\}_{\boldsymbol{y}}^{f c}\right.\right.
\end{aligned}
$$

Given a tuple of formulas $\Gamma=A_{1}, \ldots, A_{n}$, we write $\left\{[\Gamma\}_{y_{1}, \ldots, y_{n}}^{x_{1}, \ldots, x_{n}}\right.$ as an abbreviation for $\left\{\left\{A_{1}\right\}_{y_{1}}^{x_{1}}, \ldots,\left\{\left\{A_{n}\right\}_{y_{n}}^{x_{n}}\right.\right.$, assuming $A_{i}$ has interpretation $\{\{A\}\}_{y_{i}}^{x_{i}}$.

If $A$ has interpretation $\left\{\{A\}_{y}^{x}\right.$ we call $\boldsymbol{x}$ the witnesses of $A$, and $\boldsymbol{y}$ the counterwitnesses. We say that a formula $A$ has no computational content if its interpretation is $\left\{\{A\}_{\varepsilon}^{\varepsilon}\right.$, i.e. if the tuples of witnesses and counter-witnesses are both empty. We often omit $\varepsilon$, writing, for instance, $\left\{\{A\}_{\varepsilon}^{x}\right.$ as simply $\{\{A\}\}^{x}$, or $\{\{A\}\}_{y}^{\varepsilon}$ as $\{\{A\}\}_{y}$. 


\subsection{Monotonicity property for $\mathbb{B}$}

Assumption (A5) ensures that we have a term $\mathrm{m}_{\rho}^{\mathbb{B}}$ which is in some sense "bounds" the if-then-else term constructor, i.e. if $z<^{\mathbb{B}} b$ and $\boldsymbol{x}<^{\rho}$ if $_{\rho}\left(z, a_{1}, a_{2}\right)$ then $\boldsymbol{x}<^{\rho} \mathrm{m}_{\rho}^{\mathbb{B}}\left(b, a_{1}, a_{2}\right)$. We can lift this property to arbitrary formulas as follows:

Definition 2.6. For each formula A, define the tuple of terms

$$
\mathrm{m}_{A}^{\mathbb{B}}: \operatorname{wt}(\mathbb{B}) \rightarrow \tau_{A}^{+} \rightarrow \tau_{A}^{+} \rightarrow \tau_{A}^{+}
$$

by induction on A, as follows:

$$
\begin{array}{ll}
\mathrm{m}_{A \wedge B}^{\mathbb{B}}\left(b, \boldsymbol{x}_{1}, \boldsymbol{v}_{1}, \boldsymbol{x}_{2}, \boldsymbol{v}_{2}\right) & =\mathrm{m}_{A}^{\mathbb{B}}\left(b, \boldsymbol{x}_{1}, \boldsymbol{x}_{2}\right), \mathrm{m}_{B}^{\mathbb{B}}\left(b, \boldsymbol{v}_{1}, \boldsymbol{v}_{2}\right) \\
\mathrm{m}_{A \vee B}^{\mathbb{B}}\left(b, b_{1}, \boldsymbol{x}_{1}, \boldsymbol{v}_{1}, b_{2}, \boldsymbol{x}_{2}, \boldsymbol{v}_{2}\right) & =\mathrm{m}_{\mathbb{B}}^{\mathbb{B}}\left(b, b_{1}, b_{2}\right), \mathrm{m}_{A}^{\mathbb{B}}\left(b, \boldsymbol{x}_{1}, \boldsymbol{x}_{2}\right), \mathrm{m}_{B}^{\mathbb{B}}\left(b, \boldsymbol{v}_{1}, \boldsymbol{v}_{2}\right) \\
\mathrm{m}_{A \rightarrow B}^{\mathbb{B}}\left(b, \boldsymbol{f}_{1}, \boldsymbol{g}_{1}, \boldsymbol{f}_{2}, \boldsymbol{g}_{2}\right) & =\lambda \boldsymbol{x}^{\tau_{A}^{+}} \cdot \mathrm{m}_{B}^{\mathbb{B}}\left(b, \boldsymbol{f}_{1} \boldsymbol{x}, \boldsymbol{f}_{2} \boldsymbol{x}\right), \lambda \boldsymbol{x}^{\tau_{A}^{+}} \lambda \boldsymbol{w}^{\tau_{B}^{-}} \cdot \boldsymbol{g}_{1} \boldsymbol{x} \boldsymbol{w} \sqcup \boldsymbol{g}_{2} \boldsymbol{x} \boldsymbol{w} \\
\mathrm{m}_{\exists z^{\rho} A}^{\mathbb{B}}\left(b, a_{1}, \boldsymbol{x}_{1}, a_{2}, \boldsymbol{x}_{2}\right) & =\mathrm{m}_{\rho}^{\mathbb{B}}\left(b, a_{1}, a_{2}\right), \mathrm{m}_{A}^{\mathbb{B}}\left(b, \boldsymbol{x}_{1}, \boldsymbol{x}_{2}\right) \\
\mathrm{m}_{\forall z^{\rho} A}^{\mathbb{B}}\left(b, \boldsymbol{f}_{1}, \boldsymbol{f}_{2}\right) & =\lambda a^{\mathrm{wt}(\rho)} \cdot \mathrm{m}_{A}^{\mathbb{B}}\left(b, \boldsymbol{f}_{1} a, \boldsymbol{f}_{2} a\right)
\end{array}
$$

where $\mathrm{m}_{\rho}^{\mathbb{B}}$ is the term assumed to exist in (A5).

Lemma 2.7 (Monotonicity lemma for $\mathbb{B})$. Under assumption (A5), for each formula A, we have:

(i) $\vdash_{\mathcal{A}_{\mathrm{t}}} \mathrm{W}_{\mathrm{wt}(\mathbb{B}) \rightarrow \tau_{A}^{+} \rightarrow \tau_{A}^{+} \rightarrow \tau_{A}^{+}}\left(\mathrm{m}_{A}^{\mathbb{B}}\right)$

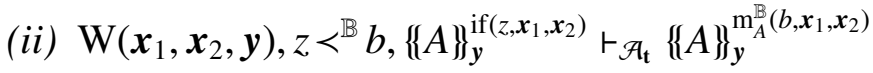

Proof. By induction on the complexity of the formula $A$. Point $(i)$ follows directly from assumptions (A5) $(i)$ and $\left(\mathbf{C}_{\sqcup}\right)$, and Lemma 2.1. As for point (ii), the cases where $A$ is an atomic formula, a conjunction, or a universal quantifier are easy to verify. Let us check the cases of existential quantifier, disjunction and implication.

Existential quantifier. Suppose $A \equiv \exists w^{\rho} B$. By induction hypothesis we have

$$
\mathrm{W}\left(\boldsymbol{x}_{1}, \boldsymbol{x}_{2}, \boldsymbol{y}\right), z<^{\mathbb{B}} b,\{\{B\}\}_{\boldsymbol{y}}^{\mathrm{if}\left(z, \boldsymbol{x}_{1}, \boldsymbol{x}_{2}\right)} \vdash \mathcal{A}_{\mathbf{t}}\{\{B\}\}_{y}^{\mathrm{m}_{A}^{\mathbb{B}}\left(b, \boldsymbol{x}_{1}, \boldsymbol{x}_{2}\right)}
$$

By $\left(\mathbf{Q}_{1}\right)$ we have

$$
\mathrm{W}\left(\boldsymbol{x}_{1}, \boldsymbol{x}_{2}, \boldsymbol{y}\right), z \prec^{\mathbb{B}} b, \forall \boldsymbol{y}^{\prime} \sqsubset \boldsymbol{y}\{\{B\}\}_{\boldsymbol{y}^{\prime}}^{\mathrm{if}\left(z, \boldsymbol{x}_{1}, \boldsymbol{x}_{2}\right)} \vdash_{\mathcal{A}_{\mathbf{t}}} \forall \boldsymbol{y}^{\prime} \sqsubset \boldsymbol{y}\{\{B\}\}_{\boldsymbol{y}^{\prime}}^{\mathrm{m}_{A}^{\mathbb{B}}\left(b, \boldsymbol{x}_{1}, \boldsymbol{x}_{2}\right)}
$$

By (A5) (ii), we get, assuming $\mathrm{W}\left(\boldsymbol{x}_{1}, \boldsymbol{x}_{2}, \boldsymbol{y}\right)$,

$z \prec^{\mathbb{B}} b, w \prec^{\rho}$ if $_{\rho}\left(z, a_{1}, a_{2}\right) \wedge \forall \boldsymbol{y}^{\prime} \sqsubset \boldsymbol{y}\left\{[B\}_{\boldsymbol{y}^{\prime}}^{\mathrm{if}\left(z, \boldsymbol{x}_{1}, \boldsymbol{x}_{2}\right)} \vdash \mathcal{A}_{\mathbf{t}} w \prec^{\rho} \mathrm{m}_{\rho}^{\mathbb{B}}\left(b, a_{1}, a_{2}\right) \wedge \forall \boldsymbol{y}^{\prime} \sqsubset \boldsymbol{y}\left\{\{B\}_{\boldsymbol{y}^{\prime}}^{\mathrm{m}_{A}^{\mathbb{B}}\left(b, \boldsymbol{x}_{1}, \boldsymbol{x}_{2}\right)}\right.\right.$ 
and hence, assuming $\mathrm{W}\left(a_{1}, \boldsymbol{x}_{1}, a_{2}, \boldsymbol{x}_{2}, \boldsymbol{y}\right)$,

$z \prec^{\mathbb{B}} b, \exists w \prec^{\rho}$ if $_{\rho}\left(z, a_{1}, a_{2}\right) \forall \boldsymbol{y}^{\prime} \sqsubset \boldsymbol{y}\left\{\{B\}_{\boldsymbol{y}^{\prime}}^{\mathrm{if}\left(z, \boldsymbol{x}_{1}, \boldsymbol{x}_{2}\right)} \vdash_{\mathcal{A}_{\mathbf{t}}} \exists w \prec^{\rho} \mathrm{m}_{\rho}^{\mathbb{B}}\left(b, a_{1}, a_{2}\right) \forall \boldsymbol{y}^{\prime} \sqsubset \boldsymbol{y}\{\{B\}\}_{\boldsymbol{y}^{\prime}}^{\mathrm{m}_{A}^{\mathbb{B}}\left(b, \boldsymbol{x}_{1}, \boldsymbol{x}_{2}\right)}\right.$

which by Definitions 2.5 and 2.6 is

$$
z \prec^{\mathbb{B}} b,\left\{\left\{\exists w^{\rho} B\right\}\right\}_{\boldsymbol{y}}^{\mathrm{if}\left(z, a_{1}, \boldsymbol{x}_{1}, a_{2}, \boldsymbol{x}_{2}\right)} \vdash \mathcal{A}_{\mathbf{t}}\left\{\left\{\exists w^{\rho} \boldsymbol{B}\right\}\right\}_{\boldsymbol{y}}^{\mathrm{m}_{\exists w}^{\mathbb{B}} \rho_{A}\left(b, a_{1}, \boldsymbol{x}_{1}, a_{2}, \boldsymbol{x}_{2}\right)}
$$

Disjunction. The case of disjunction is similar to the existential quantifier above, and also uses the assumption (A5) for $\rho=\mathbb{B}$.

Implication. Assume $\mathrm{W}\left(\boldsymbol{f}_{1}, \boldsymbol{g}_{1}, \boldsymbol{f}_{2}, \boldsymbol{g}_{2}, \boldsymbol{x}, \boldsymbol{w}\right)$ and $z \prec^{\mathbb{B}} b$. Noting that

(*) $\forall y \sqsubset_{\tau_{A}^{-}} g_{1} x w\left\{\{A\}_{y}^{x}, \forall y \sqsubset \tau_{\tau_{A}^{-}} g_{2} x w\left\{\{A\}_{y}^{x} \vdash \forall y \sqsubset_{\tau_{A}^{-}}\right.\right.$if $\left(z, g_{1} x w, g_{2} x w\right)\left\{\{A\}_{y}^{x}\right.$

we have

$$
\begin{aligned}
& \{(A \rightarrow B)\}\}_{x, w}^{\mathrm{if}\left(z, f_{1}, \boldsymbol{g}_{1}, f_{2}, \boldsymbol{g}_{2}\right)} \\
& \equiv \forall \boldsymbol{y} \sqsubset_{\tau_{A}^{-}} \text {if }\left(z, g_{1} x w, g_{2} x w\right)\left\{\{ A \} _ { y } ^ { x } \rightarrow \left\{\{B\}_{w}^{\mathrm{if}\left(z, f_{1} x, f_{2} x\right)}\right.\right. \\
& \stackrel{(*)}{\Rightarrow} \forall y \sqsubset_{\tau_{A}^{-}} g_{1} x w\left\{\{ A \} _ { y } ^ { x } \wedge \forall y \sqsubset _ { \tau _ { A } ^ { - } } g _ { 2 } x w \left\{\{ A \} _ { y } ^ { x } \rightarrow \left\{\{B\}_{w}^{\text {if }\left(z, f_{1} x, f_{2} x\right)}\right.\right.\right. \\
& \stackrel{\left(\mathrm{C}_{\sqcup}\right)}{\Rightarrow} \quad \forall \boldsymbol{y} \sqsubset_{\tau_{A}^{-}} \boldsymbol{g}_{1} \boldsymbol{x w} \sqcup \boldsymbol{g}_{2} \boldsymbol{x w}\left\{\{ A \} _ { \boldsymbol { y } } ^ { \boldsymbol { x } } \rightarrow \left\{\{B\}_{\boldsymbol{w}}^{\mathrm{if}\left(z, f_{1} x, f_{2} x\right)}\right.\right. \\
& \stackrel{(\mathrm{IH})}{\Rightarrow} \forall \boldsymbol{y} \sqsubset_{\tau_{A}^{-}} \boldsymbol{g}_{1} \boldsymbol{x} \boldsymbol{w} \sqcup \boldsymbol{g}_{2} \boldsymbol{x w}\left\{\{ A \} _ { \boldsymbol { y } } ^ { \boldsymbol { x } } \rightarrow \left\{\{B\}_{\boldsymbol{w}^{\prime}}^{\mathrm{m}_{B}^{\mathrm{B}}\left(b, f_{1} \boldsymbol{x}, f_{2} x\right)}\right.\right. \\
& \equiv\{(A \rightarrow B)\}_{x, \boldsymbol{w}}^{\mathrm{m}_{A \rightarrow B}^{\mathrm{B}}\left(b, f_{1}, \boldsymbol{g}_{1}, f_{2}, \boldsymbol{g}_{2}\right)}
\end{aligned}
$$

since the assumptions imply $\mathrm{W}\left(\boldsymbol{f}_{1} \boldsymbol{x}, \boldsymbol{f}_{2} \boldsymbol{x}, \boldsymbol{w}\right)$ - needed for the (IH).

\subsection{Monotonicity property for $\mathbb{N}$}

As with the booleans above, we can also lift assumption (A6) to arbitrary formulas as follows:

Definition 2.8. For each intuitionistic formula A, define the tuple of terms

$$
\mathrm{m}_{A}^{\mathbb{N}}:\left(\mathbb{N} \rightarrow \tau_{A}^{+}\right) \rightarrow \operatorname{wt}(\mathbb{N}) \rightarrow \tau_{A}^{+}
$$

by induction on $A$, as follows:

$$
\begin{array}{ll}
\mathrm{m}_{A \wedge B}^{\mathbb{N}}\left(\boldsymbol{f}_{A}, \boldsymbol{f}_{B}\right)(a) & =\mathrm{m}_{A}^{\mathbb{N}}\left(\boldsymbol{f}_{A}\right)(a), \mathrm{m}_{B}^{\mathbb{N}}\left(\boldsymbol{f}_{B}\right)(a) \\
\mathrm{m}_{A \vee B}^{\mathbb{N}}\left(b_{A}, \boldsymbol{f}_{A}, b_{B}, \boldsymbol{f}_{B}\right)(a) & =\mathrm{m}_{\mathbb{B}}^{\mathbb{N}}\left(b_{A} a, b_{B} a\right)(a), \mathrm{m}_{A}^{\mathbb{N}}\left(\boldsymbol{f}_{A}\right)(a), \mathrm{m}_{B}^{\mathbb{N}}\left(\boldsymbol{f}_{B}\right)(a) \\
\mathrm{m}_{A \rightarrow B}^{\mathbb{N}}(\boldsymbol{f}, \boldsymbol{g})(a) & =\lambda \boldsymbol{x}^{\boldsymbol{\tau}_{A}^{+}} \cdot \mathrm{m}_{B}^{\mathbb{N}}(\lambda n \cdot \boldsymbol{f} n \boldsymbol{x})(a) ; \lambda \boldsymbol{x}^{\tau_{A}^{+}}, \boldsymbol{w}^{\boldsymbol{\tau}_{B}^{-}} \cdot \boldsymbol{g}^{\sqcup}(N a) \boldsymbol{x} \boldsymbol{w} \\
\mathrm{m}_{\exists x_{A} A}^{\mathbb{N}}\left(h^{\mathbb{N} \rightarrow \operatorname{wt}(\rho)}, \boldsymbol{f}^{\mathbb{N} \rightarrow \boldsymbol{\tau}_{A}^{+}}\right)(a) & =\mathrm{m}_{\rho}^{\mathbb{N}}(h)(a), \mathrm{m}_{A}^{\mathbb{N}}(\boldsymbol{f})(a) \\
\mathrm{m}_{\forall x^{\rho} A}^{\mathbb{N}}\left(\boldsymbol{f}^{\mathbb{N} \rightarrow \operatorname{wt}(\rho) \rightarrow \tau_{A}^{+}}\right)(a) & =\lambda b^{\operatorname{wt}(\rho)} \mathrm{m}_{A}^{\mathbb{N}}(\lambda n \cdot \boldsymbol{f} n b)(a)
\end{array}
$$


where $\mathrm{m}_{\rho}^{\mathbb{N}}$ and $N$ are the terms assumed to exist in (A6), and $\mathbf{g}^{\sqcup}$ is defined recursively as

$$
\boldsymbol{g}^{\sqcup} n \boldsymbol{x} \boldsymbol{w}= \begin{cases}\boldsymbol{g} 0 \boldsymbol{x} \boldsymbol{w} & \text { if } n=0 \\ \boldsymbol{g} n \boldsymbol{x} \boldsymbol{w} \sqcup \boldsymbol{g}^{\sqcup}(n-1) \boldsymbol{x} \boldsymbol{w} & \text { if } n>0\end{cases}
$$

Lemma 2.9 (Monotonicity lemma for $\mathbb{N}$ ). Under assumption (A6), for each formula A, we have:

(i) $\vdash_{\mathcal{A}_{\mathbf{t}}} \mathrm{W}_{\left(\mathbb{N} \rightarrow \tau_{A}^{+}\right) \rightarrow \mathrm{wt}(\mathbb{N}) \rightarrow \tau_{A}^{+}}\left(\mathrm{m}_{P}^{\mathbb{N}}\right)$

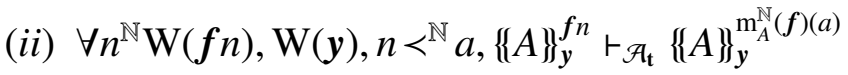

Proof. The proof is very similar to that of the monotonicity lemma for $\mathbb{B}$ (Lemma 2.7). In the case of implication we first need to prove

$$
\text { (*) } \forall \boldsymbol{y} \sqsubset_{\tau_{A}^{-}} \boldsymbol{g}^{\sqcup}(N a) \boldsymbol{x} \boldsymbol{w}\left\{\{A\}_{\boldsymbol{y}}^{\boldsymbol{x}}, n \prec^{\mathbb{N}} a \vdash_{\mathcal{A}_{\mathbf{t}}} \forall \boldsymbol{y} \sqsubset_{\tau_{A}^{-}} \boldsymbol{g} n \boldsymbol{x} \boldsymbol{w}\{\{A\}\}_{\boldsymbol{y}}^{\boldsymbol{x}}\right.
$$

which we can do by first proving, using induction on $k$ and then taking $k=N a$,

$$
\forall \boldsymbol{y} \sqsubset_{\tau_{A}^{-}} \boldsymbol{g}^{\sqcup} k \boldsymbol{x} \boldsymbol{w}\left\{\{A\}_{y}^{\boldsymbol{x}}, n \leq k \vdash \mathcal{A}_{\mathrm{t}} \forall \boldsymbol{y} \sqsubset_{\tau_{A}^{-}} \boldsymbol{g} n \boldsymbol{x} \boldsymbol{w}\left\{\{A\}_{\boldsymbol{y}}^{\boldsymbol{x}}\right.\right.
$$

The above also uses applications of $\left(\mathbf{C}_{\sqcup}\right)$ for the induction step. Hence, assuming $\forall n^{\mathbb{N}} \mathrm{W}(\boldsymbol{f} n, \boldsymbol{g} n)$ and $\mathrm{W}(\boldsymbol{x}, \boldsymbol{w})$ and $\left(n \prec^{\mathbb{N}} a\right)$ we have

$$
\begin{aligned}
& \left\{\{ A \rightarrow B \} _ { x , w } ^ { f n , g n } \equiv \forall \boldsymbol { y } \sqsubset _ { \tau _ { A } ^ { - } } \boldsymbol { g n x \boldsymbol { x } } \{ \{ A \} \} _ { y } ^ { x } \rightarrow \left\{\{B\}_{w}^{f n x}\right.\right. \\
& \stackrel{(*)}{\Rightarrow} \quad \forall \boldsymbol{y} \sqsubset_{\tau_{A}^{-}} \boldsymbol{g}^{\sqcup}(N a) \boldsymbol{x} \boldsymbol{w}\left\{\{ A \} _ { \boldsymbol { y } } ^ { \boldsymbol { x } } \rightarrow \left\{\{B\}_{\boldsymbol{w}}^{(\lambda n . f n x)(n)}\right.\right. \\
& \stackrel{(\mathrm{IH})}{\Rightarrow} \forall \boldsymbol{y} \sqsubset_{\tau_{A}^{-}} \boldsymbol{g}^{\sqcup}(N a) \boldsymbol{x} \boldsymbol{w}\left\{\{ A \} _ { \boldsymbol { y } } ^ { \boldsymbol { x } } \rightarrow \left\{\{B\}_{w^{\mathrm{m}}}^{\mathrm{m}_{B}^{\mathrm{N}}(\lambda n . f n \boldsymbol{x})(a)}\right.\right. \\
& \equiv\left\{\{A \rightarrow B\}_{\boldsymbol{x}, \boldsymbol{w}}^{\mathrm{m}_{A \rightarrow B}^{\mathrm{N}}(f, \boldsymbol{g})(a)}\right.
\end{aligned}
$$

since the assumptions imply $\forall n^{\mathbb{N}} \mathrm{W}(\boldsymbol{f} n \boldsymbol{x})$ - needed for the (IH).

\subsection{Soundness}

We are now ready to present the (parametrised) soundness of the parametrised functional interpretation (Definition 2.5).

Definition 2.10 (Witnessable sequents). A sequent $\Gamma\left[\boldsymbol{m}^{\tau}\right] \vdash A\left[\boldsymbol{m}^{\tau}\right]$ of WE-HA ${ }^{\omega}$ (with free variables $\boldsymbol{m}^{\tau}$ ) is said to be $\{\{\}\}$-witnessable in the target theory $\mathcal{A}_{\mathbf{t}}$ if there are tuples of closed terms $\boldsymbol{\gamma}$, a of $\mathcal{A}_{\mathbf{t}}$ such that

(i) $\vdash_{\mathcal{A}_{\mathrm{t}}} \mathrm{W}_{\tau_{\Gamma}^{+} \rightarrow \tau_{A}^{-} \rightarrow \operatorname{wt}(\tau) \rightarrow \operatorname{bt}\left(\tau_{\Gamma}^{-}\right)}(\boldsymbol{\gamma})$ and $\vdash_{\mathcal{A}_{\mathbf{t}}} \mathrm{W}_{\tau_{\Gamma}^{+} \rightarrow \operatorname{wt}(\tau) \rightarrow \tau_{A}^{+}}(\boldsymbol{a})$ 
(ii) $\mathrm{W}_{\tau_{\Gamma}^{+}, \tau_{A}^{-}}(\boldsymbol{v}, \boldsymbol{y}), \boldsymbol{m}<^{\tau} \tilde{\boldsymbol{m}}, \forall \boldsymbol{w} \sqsubset_{\tau_{\Gamma}^{-}} \boldsymbol{\gamma} \boldsymbol{v y} \tilde{\boldsymbol{m}}\left\{[\Gamma[\boldsymbol{m}]\}_{w}^{v} \vdash \mathcal{A}_{\mathrm{t}}\left\{\{A[\boldsymbol{m}]\}_{\boldsymbol{y}}^{a v \tilde{m}}\right.\right.$

Definition 2.11 (Sound \{\{\}\}-interpretation). An instantiation of the \{\{\}\}-interpretation is said to be sound if the provable sequents of $\mathbf{W E}-\mathbf{H} \mathbf{A}^{\omega}$ are $\{\{\}$-witnessable in the target theory $\mathcal{A}_{\mathbf{t}}$.

Theorem 2.12 (Soundness of the $\{\{\}\}$-interpretation). Assume a fixed choice of the parameters for a theory $\mathcal{A}_{\mathbf{t}}$. Let $C$ be the set of formulas $\left\{\{A\}_{y}^{x}\right.$, for formulas $A$ of WE-HA $^{\omega}$. If this choice of parameters is $C$-adequate for $\mathcal{A}_{\mathbf{t}}($ Def. 2.2$)$ then this instance of the parametrised interpretation (Def. 2.5) is sound (Def. 2.11).

Proof. We must show that the provable sequents $\Gamma[\boldsymbol{m}] \vdash A[\boldsymbol{m}]$ of WE-HA ${ }^{\omega}$ are $\left\{\{\}\right.$-witnessable in $\mathcal{A}_{\mathbf{t}}$. We do this by induction on the WE-HA ${ }^{\omega}$ derivation of $\Gamma[\boldsymbol{m}] \vdash A[\boldsymbol{m}]$. The free-variables $\boldsymbol{m}$ only play an important role in the treatment of the quantifiers. Therefore, in the treatment of the logical rules we will w.l.o.g. assume that $\Gamma$ and $A$ have no free variables.

The non-logical axioms of WE-HA ${ }^{\omega}$ (except for induction) and the weak extensionality rule are easily seen to be witnessable since atomic formulas have empty "computational content". We treat the induction rule as the last case in this proof. Let us first consider each of the logical rules (see Table 1) and show that they turn witnessable premisses into witnessable conclusions. In each case we need to prove points $(i)$ and (ii) of Definition 2.10. Point $(i)$, however, will follow directly from the induction hypothesis and Lemma 2.1, since the terms witnessing the conclusion of each rule will be built from the terms witnessing the premise via simple $\lambda$-term constructions (definable from $\mathrm{S}$ and $\mathrm{K}$ ). Therefore, we will focus our attention on proving point (ii).

$C u t$. By induction hypothesis we have closed terms $\boldsymbol{a}_{0}, \boldsymbol{a}_{1}, \boldsymbol{\gamma}, \boldsymbol{\delta}, \boldsymbol{b}$ such that

$$
\begin{aligned}
\left(\mathrm{IH}_{(i)}\right) & \vdash \mathrm{W}\left(\boldsymbol{a}_{0}\right) \text { and } \vdash \mathrm{W}(\boldsymbol{\gamma}) \text { and } \vdash \mathrm{W}(\boldsymbol{b}) \text { and } \vdash \mathrm{W}(\boldsymbol{\delta}) \text { and } \vdash \mathrm{W}\left(\boldsymbol{a}_{1}\right) \\
\left(\mathrm{IH}_{(i i)}\right) & \mathrm{W}(\boldsymbol{u}, \boldsymbol{y}), \forall \boldsymbol{w}^{\prime} \sqsubset_{\tau_{\Gamma}^{-}} \boldsymbol{\gamma} \boldsymbol{u} \boldsymbol{y}\left\{\{ \Gamma \} _ { w ^ { \prime } } ^ { u } \vdash \left\{\{A\}_{\boldsymbol{y}}^{\boldsymbol{a}_{0} \boldsymbol{u}}\right.\right. \text { and } \\
& \mathrm{W}(\boldsymbol{v}, \boldsymbol{x}, \boldsymbol{w}), \forall \boldsymbol{w}^{\prime \prime} \sqsubset_{\tau_{\Delta}^{-}} \boldsymbol{\delta} \boldsymbol{v} \boldsymbol{x} \boldsymbol{w}\left\{\{\Delta\}_{\boldsymbol{w}^{\prime \prime}}^{v}, \forall \boldsymbol{y} \sqsubset_{\tau_{A}^{-}} \boldsymbol{a}_{1} \boldsymbol{v} \boldsymbol{x} \boldsymbol{w}\{\{A\}\}_{\boldsymbol{y}}^{\boldsymbol{x}} \vdash\left\{\{B\}_{\boldsymbol{w}}^{b \boldsymbol{v} x}\right.\right.
\end{aligned}
$$

Let $\tilde{\boldsymbol{a}}: \equiv \lambda \boldsymbol{u}, \boldsymbol{v}, \boldsymbol{w} \cdot \boldsymbol{a}_{1} \boldsymbol{v}\left(\boldsymbol{a}_{0} \boldsymbol{u}\right) \boldsymbol{w}$ and $\tilde{\boldsymbol{b}}: \equiv \lambda \boldsymbol{u}, \boldsymbol{v} \cdot \boldsymbol{b} \boldsymbol{v}\left(\boldsymbol{a}_{0} \boldsymbol{u}\right)$ and $\tilde{\boldsymbol{\gamma}}: \equiv \lambda \boldsymbol{u}, \boldsymbol{v}, \boldsymbol{w} \cdot(\boldsymbol{\gamma} \boldsymbol{u}) \circ$ $(\tilde{\boldsymbol{a}} \boldsymbol{u v} \boldsymbol{w})$ and $\tilde{\boldsymbol{\delta}}: \equiv \lambda \boldsymbol{u}, \boldsymbol{v}, \boldsymbol{w} . \boldsymbol{\delta} \boldsymbol{v}\left(\boldsymbol{a}_{0} \boldsymbol{u}\right) \boldsymbol{w}$. Using the induction hypothesis $\left(\mathrm{IH}_{(i)}\right)$ and 
$\left(\mathrm{IH}_{(i i)}\right)$ we have

$$
\begin{aligned}
& \overline{\mathrm{W}(\boldsymbol{u}, \boldsymbol{y}), \forall \boldsymbol{w}^{\prime} \sqsubset_{\tau_{\Gamma}^{-}} \gamma \boldsymbol{u} \boldsymbol{y}\left\{[ \Gamma \} _ { \boldsymbol { w } ^ { \prime } } ^ { \boldsymbol { u } } \vdash \left\{\{A\}_{\boldsymbol{y}}^{\boldsymbol{a}_{0} \boldsymbol{u}}\right.\right.}\left(\mathrm{IH}_{(i i)}\right)
\end{aligned}
$$

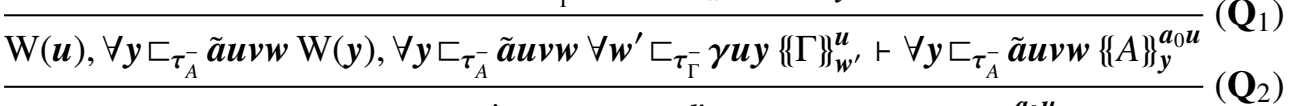

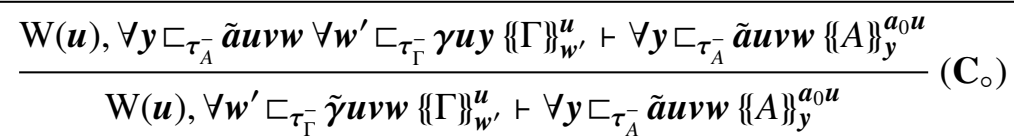

Using the above (in the cut step) and $\mathrm{W}(\boldsymbol{u}) \vdash \mathrm{W}\left(\boldsymbol{a}_{0} \boldsymbol{u}\right)$, we then have

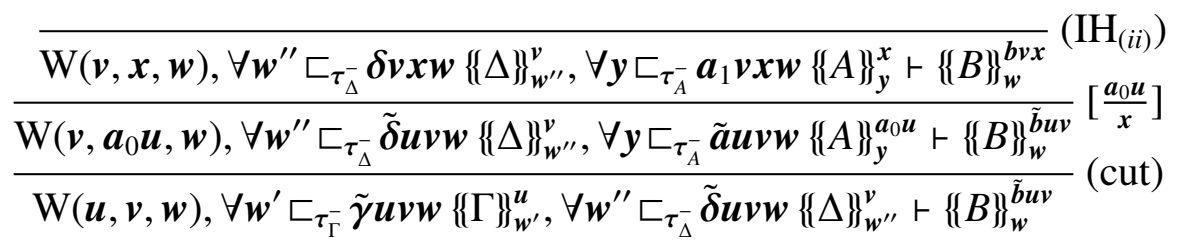

$(\wedge \mathrm{R})$. Direct, from the induction hypothesis.

For the sake of presentation, in the cases below we may omit the contexts $\Gamma$ and $\Delta$ whenever they do not play an important role in the treatment of the rule.

$(\wedge \mathrm{L})$. By the induction hypothesis we have closed terms $\boldsymbol{a}, \boldsymbol{b}, \boldsymbol{c}$ in $\mathrm{W}$ such that

(IH) $\mathrm{W}(\boldsymbol{x}, \boldsymbol{v}, \boldsymbol{w}), \forall \boldsymbol{y} \sqsubset_{\tau_{A}^{-}} \operatorname{axvw}\left\{\{A\}_{y}^{x}, \forall \boldsymbol{w}^{\prime} \sqsubset_{\tau_{B}^{-}} \boldsymbol{b x v w}\left\{\{B\}_{w^{\prime}}^{v} \vdash\left\{[C\}_{w}^{c x v}\right.\right.\right.$

We claim that the terms $\boldsymbol{\gamma}, \boldsymbol{a}, \boldsymbol{b}$ and $\boldsymbol{c}$ witness the conclusion of the rule. We have

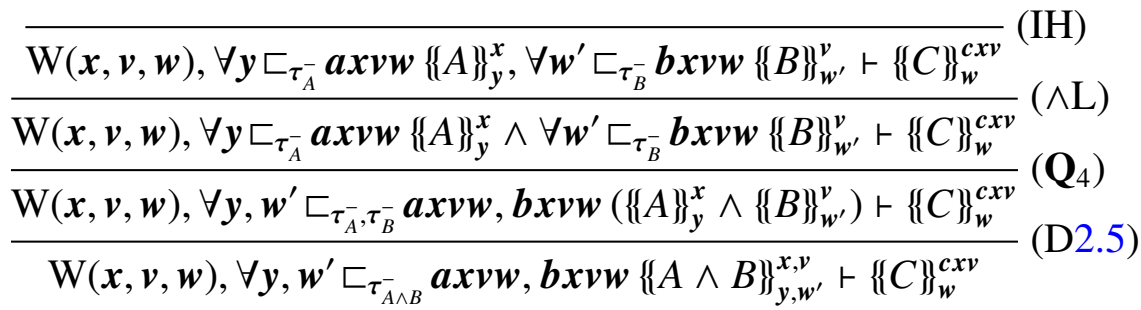

$(\mathrm{VL})$. By induction hypothesis we have

1. $\mathrm{W}(\boldsymbol{x}, \boldsymbol{u}), \forall \boldsymbol{y} \sqsubset_{\tau_{A}^{-}} \boldsymbol{a x \boldsymbol { x }}\left\{\{A\}_{\boldsymbol{y}}^{\boldsymbol{x}} \vdash\left\{[C\}_{\boldsymbol{u}}^{\boldsymbol{c}_{A} \boldsymbol{x}}\right.\right.$, for closed terms $\boldsymbol{a}$ and $\boldsymbol{c}_{A}$ in W

2. $\mathrm{W}(\boldsymbol{v}, \boldsymbol{u}), \forall \boldsymbol{w}{\check{\tau_{B}^{-}}}^{-} \boldsymbol{b} \boldsymbol{v} \boldsymbol{u}\left\{[B\}_{\boldsymbol{w}}^{v} \vdash\left\{\{C\}_{\boldsymbol{u}}^{\boldsymbol{c}_{B} \boldsymbol{v}}\right.\right.$, for closed terms $\boldsymbol{b}$ and $\boldsymbol{c}_{B}$ in $\mathrm{W}$

The above implies

1. $\mathrm{W}(\boldsymbol{x}, \boldsymbol{u}), \forall \boldsymbol{y} \sqsubset_{\tau_{A}^{-}} \boldsymbol{a x u}\{\{A\}\}_{\boldsymbol{y}}^{\boldsymbol{x}}, z<^{\mathbb{B}} b, z=\mathrm{T} \vdash\{\{C\}\}_{\boldsymbol{u}}^{\mathrm{if}\left(z, \boldsymbol{c}_{A} x, c_{B} v\right)}$

2. $\mathrm{W}(\boldsymbol{v}, \boldsymbol{u}), \forall \boldsymbol{w} \sqsubset_{\tau_{B}^{-}} \boldsymbol{b} v \boldsymbol{u}\left\{\{B\}_{w}^{v}, z<^{\mathbb{B}} b, z=\mathrm{F} \vdash\left\{\{C\}_{\boldsymbol{u}}^{\mathrm{if}\left(z, c_{A} x, c_{B} v\right)}\right.\right.$ 
By Lemma 2.7

1. $\mathrm{W}(\boldsymbol{x}, \boldsymbol{v}, \boldsymbol{u}), \forall \boldsymbol{y} \sqsubset_{\tau_{A}^{-}} \boldsymbol{a x} \boldsymbol{u}\left\{\{A\}_{\boldsymbol{y}}^{\boldsymbol{x}}, z \prec^{\mathbb{B}} b, z=\mathrm{T} \vdash\left\{\{C\}_{\boldsymbol{u}}^{\mathrm{m}_{C}^{\mathbb{B}}\left(b, \boldsymbol{c}_{A} \boldsymbol{x}, \boldsymbol{c}_{B} v\right)}\right.\right.$

2. $\mathrm{W}(\boldsymbol{x}, \boldsymbol{v}, \boldsymbol{u}), \forall \boldsymbol{w} \sqsubset_{\tau_{B}^{-}} \boldsymbol{b} \boldsymbol{v} \boldsymbol{u}\left\{\{B\}_{\boldsymbol{w}}^{\boldsymbol{v}}, z \prec^{\mathbb{B}} b, z=\mathrm{F} \vdash\left\{\{C\}_{\boldsymbol{u}}^{\mathrm{m}_{C}^{\mathbb{B}}\left(b, \boldsymbol{c}_{A} \boldsymbol{x}, \boldsymbol{c}_{B} \boldsymbol{v}\right)}\right.\right.$

By (VL), we get

$$
\begin{aligned}
& \mathrm{W}(\boldsymbol{x}, \boldsymbol{v}, \boldsymbol{u}), \\
& z \prec^{\mathbb{B}} b,\left(\left(z=\mathrm{T} \wedge \forall \boldsymbol{y} \sqsubset_{\tau_{A}^{-}} \boldsymbol{a x u}\left\{\{A\}_{\boldsymbol{y}}^{\boldsymbol{x}}\right) \vee\left(z=\mathrm{F} \wedge \forall \boldsymbol{w} \sqsubset_{\boldsymbol{\tau}_{B}^{-}} \boldsymbol{b v u}\{\{B\}\}_{\boldsymbol{w}}^{\boldsymbol{v}}\right)\right)\right. \\
& \vdash\left\{\{C\}_{\boldsymbol{u}_{C}^{\mathrm{B}}}^{\mathrm{B}^{\mathbb{B}}\left(b, \boldsymbol{c}_{A} \boldsymbol{x}, \boldsymbol{c}_{B} \boldsymbol{v}\right)}\right.
\end{aligned}
$$

By $(\exists \mathrm{L})$, we get

$$
\begin{aligned}
& \mathrm{W}(\boldsymbol{x}, \boldsymbol{v}, \boldsymbol{u}), \\
& \exists z \prec^{\mathbb{B}} b\left(\left(z=\mathrm{T} \wedge \forall \boldsymbol{y} \sqsubset_{\tau_{A}^{-}} \boldsymbol{a x u}\left\{\{A\}_{\boldsymbol{y}}^{\boldsymbol{x}}\right) \vee\left(z=\mathrm{F} \wedge \forall \boldsymbol{w} \sqsubset_{\tau_{B}^{-}} \boldsymbol{b} \boldsymbol{v} \boldsymbol{u}\{\{B\}\}_{\boldsymbol{w}}^{v}\right)\right)\right. \\
& \vdash\{C C\}_{\boldsymbol{u}}^{\mathrm{m}_{C}^{\mathrm{B}}\left(b, \boldsymbol{c}_{A} \boldsymbol{x}, \boldsymbol{c}_{B} \boldsymbol{v}\right)}
\end{aligned}
$$

And hence, by $\left(\mathbf{C}_{\eta}\right)$,

$$
\begin{aligned}
& \mathrm{W}(\boldsymbol{x}, \boldsymbol{v}, \boldsymbol{u}), \\
& \forall \tilde{\boldsymbol{y}}, \tilde{\boldsymbol{w}} \sqsubset_{\mathrm{bt}\left(\tau_{A}^{-}\right), \mathrm{bt}\left(\tau_{B}^{-}\right)} \eta(\boldsymbol{a} \boldsymbol{x} \boldsymbol{u}), \eta(\boldsymbol{b} \boldsymbol{v} \boldsymbol{u}) \\
& \quad \exists z \prec^{\mathbb{B}} b\left(\left(z=\mathrm{T} \wedge \forall \boldsymbol{y} \sqsubset_{\tau_{A}^{-}} \tilde{\boldsymbol{y}}\left\{\{A\}_{\boldsymbol{y}}^{\boldsymbol{x}}\right) \vee\left(z=\mathrm{F} \wedge \forall \boldsymbol{w} \sqsubset_{\tau_{B}^{-}} \tilde{\boldsymbol{w}}\left\{\{B\}_{\boldsymbol{w}}^{\boldsymbol{v}}\right)\right)\right.\right. \\
& \quad \vdash\{C\}_{\boldsymbol{u}}^{\mathrm{m}_{C}^{\mathbb{B}}\left(b, \boldsymbol{c}_{A} \boldsymbol{x}, \boldsymbol{c}_{B} \boldsymbol{v}\right)}
\end{aligned}
$$

which by Definition 2.5 is

$$
\mathrm{W}(\boldsymbol{x}, \boldsymbol{v}, \boldsymbol{u}), \forall \tilde{\boldsymbol{y}}, \tilde{\boldsymbol{w}} \sqsubset_{\tau_{A \vee B}^{-}} \eta(\boldsymbol{a x} \boldsymbol{u}), \eta(\boldsymbol{b} v \boldsymbol{u})\left\{\{ A \vee B \} _ { \tilde { \boldsymbol { y } } , \tilde { \boldsymbol { w } } } ^ { b , \boldsymbol { x } , \boldsymbol { v } } \vdash \left\{\{C\}_{\boldsymbol{u}}^{\mathrm{m}_{C}^{\mathbb{B}}\left(b, \boldsymbol{c}_{A} \boldsymbol{x}, \boldsymbol{c}_{B} v\right)}\right.\right.
$$

$(\rightarrow \mathrm{R})$. Direct, from the induction hypothesis.

$(\rightarrow \mathrm{L})$. By the induction hypothesis we have closed terms $\boldsymbol{a}, \boldsymbol{b}, \boldsymbol{c}, \boldsymbol{\gamma}, \boldsymbol{\delta}$ such that $\left(\mathrm{IH}_{(i)}\right) \vdash \mathrm{W}(\boldsymbol{a})$ and $\vdash \mathrm{W}(\boldsymbol{b})$ and $\vdash \mathrm{W}(\boldsymbol{c})$ and $\vdash \mathrm{W}(\boldsymbol{\gamma})$ and $\vdash \mathrm{W}(\boldsymbol{\delta})$ $\left(\mathrm{IH}_{(i i)}\right) \mathrm{W}(\boldsymbol{u}, \boldsymbol{y}), \forall \boldsymbol{w}^{\prime} \sqsubset_{\tau_{\Gamma}^{-}} \gamma \boldsymbol{u} \boldsymbol{y}\left\{\{\Gamma\}_{w^{\prime}}^{\boldsymbol{u}} \vdash\{\{A\}\}_{\boldsymbol{y}}^{a \boldsymbol{u}}\right.$ and $\mathrm{W}(\boldsymbol{w}, \boldsymbol{v}, z), \forall \boldsymbol{w}^{\prime \prime} \sqsubset_{\tau_{\Delta}^{-}} \delta w \boldsymbol{w} z\{\{\Delta\}\}_{w^{\prime \prime}}^{w}, \forall \boldsymbol{w}^{\prime \prime \prime} \sqsubset_{\tau_{B}^{-}} \boldsymbol{b} w \boldsymbol{w} z\left\{\{B\}_{w^{\prime \prime \prime}}^{v} \vdash\{\{C\}\}_{z}^{c w v}\right.$ 
Using $\left(\mathrm{IH}_{(i)}\right)$ and $\left(\mathrm{IH}_{(i i)}\right)$, with $\tilde{\boldsymbol{b}}=\boldsymbol{b} \boldsymbol{w}(\boldsymbol{f}(\boldsymbol{a u})) \boldsymbol{z}$ and $\boldsymbol{d}=\boldsymbol{g}(\boldsymbol{a u}) \circ \tilde{\boldsymbol{b}}$, we have

$$
\begin{aligned}
& \overline{\mathrm{W}(\boldsymbol{u}, \boldsymbol{y}), \forall \boldsymbol{w}^{\prime} \sqsubset_{\tau_{\Gamma}^{-}} \boldsymbol{\gamma} \boldsymbol{u} \boldsymbol{y}\left\{[ \Gamma \} _ { w ^ { \prime } } ^ { u } \vdash \left\{\{A\}_{\boldsymbol{y}}^{a u}\right.\right.}\left(\mathrm{IH}_{(i i)}\right) \\
& \frac{\overline{\mathrm{W}(\boldsymbol{u}, \boldsymbol{g}), \forall \boldsymbol{y} \sqsubset_{\tau_{A}^{-}} \boldsymbol{d} \mathrm{W}(\boldsymbol{y}), \forall \boldsymbol{y} \sqsubset_{\tau_{A}^{-}} \boldsymbol{d} \forall \boldsymbol{w}^{\prime} \sqsubset_{\tau_{\Gamma}^{-}} \gamma \boldsymbol{u} \boldsymbol{y}\left\{[ \Gamma \} _ { w ^ { \prime } } ^ { \boldsymbol { u } } \vdash \forall \boldsymbol { y } \sqsubset _ { \tau _ { A } ^ { - } } \boldsymbol { d } \left\{\{A\}_{\boldsymbol{y}}^{a u}\right.\right.}\left(\mathbf{Q}_{1}\right)}{\mathrm{W}(\boldsymbol{u}, \boldsymbol{g}), \forall \boldsymbol{w}^{\prime} \sqsubset_{\tau_{\Gamma}^{-}} \gamma \boldsymbol{u} \circ \boldsymbol{d}\left\{\{ \Gamma \} _ { w ^ { \prime } } ^ { \boldsymbol { u } } \vdash \forall \boldsymbol { y } \sqsubset _ { \tau _ { A } ^ { - } } \boldsymbol { d } \left\{\{A\}_{\boldsymbol{y}}^{a u}\right.\right.}\left(\mathbf{C}_{\circ}\right)
\end{aligned}
$$

and, letting $\tilde{\boldsymbol{c}}=\boldsymbol{c w}(\boldsymbol{f}(\boldsymbol{a u}))$ and $\tilde{\delta}=\delta \boldsymbol{w}(\boldsymbol{f}(\boldsymbol{a u})) \boldsymbol{z}$

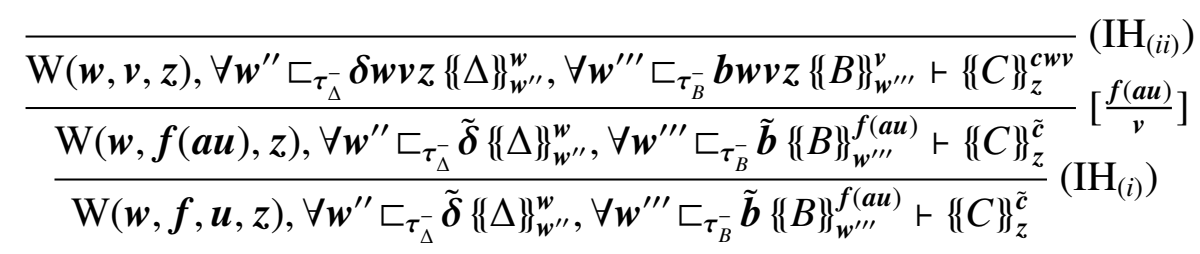

Let us call the two derivations above $\pi_{1}$ and $\pi_{2}$. Then (omitting the contexts $\forall \boldsymbol{w}^{\prime} \sqsubset_{\tau_{\Gamma}^{-}} \boldsymbol{\gamma} \boldsymbol{u} \circ \boldsymbol{d}\left\{\{\Gamma\}_{w^{\prime}}^{u}\right.$ and $\forall \boldsymbol{w}^{\prime \prime} \sqsubset_{\tau_{\Delta}^{-}} \tilde{\boldsymbol{\delta}}\{\{\Delta\}\}_{w^{\prime \prime}}^{w}$ for the sake of presentation):

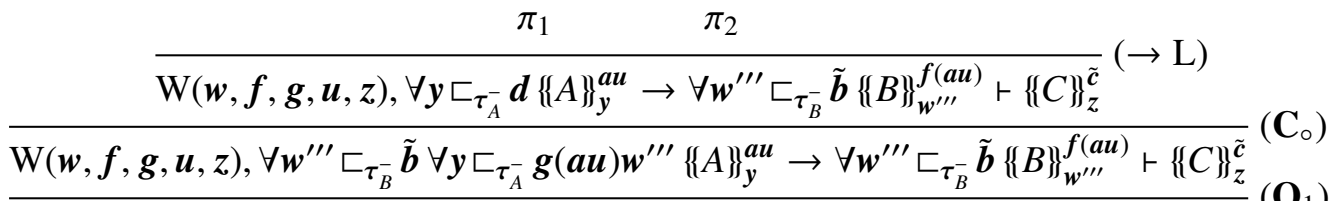

$$
\begin{aligned}
& \mathrm{W}(\boldsymbol{w}, \boldsymbol{f}, \boldsymbol{g}, \boldsymbol{u}, z), \forall \boldsymbol{w}^{\prime \prime \prime} \sqsubset_{\tau_{B}^{-}} \tilde{\boldsymbol{b}}\left(\forall \boldsymbol { y } \sqsubset _ { \tau _ { A } ^ { - } } \boldsymbol { g } ( \boldsymbol { a u } ) \boldsymbol { w } ^ { \prime \prime \prime } \left\{\{A\}_{y}^{a u} \rightarrow\left\{\{B\}_{w^{\prime \prime \prime}}^{f(a u)}\right)+\left\{\{C\}_{z}^{\tilde{c}}\right.\right.\right. \\
& \mathrm{W}(\boldsymbol{w}, \boldsymbol{f}, \boldsymbol{g}, \boldsymbol{u}, \boldsymbol{z}), \forall \boldsymbol{w}^{\prime \prime \prime} \sqsubset_{\tau_{B}^{-}} \tilde{\boldsymbol{b}}\left\{\{A \rightarrow B\}_{a u, w^{\prime \prime \prime}}^{f, \boldsymbol{g}} \vdash\{C C\}_{z}^{\tilde{c}}\right. \\
& \overline{\mathrm{W}(\boldsymbol{w}, \boldsymbol{f}, \boldsymbol{g}, \boldsymbol{u}, \boldsymbol{z}), \forall \boldsymbol{w}^{\prime \prime \prime} \sqsubset_{\boldsymbol{\tau}_{B}^{-}} \tilde{\boldsymbol{b}} \forall \boldsymbol{x} \sqsubset_{\tau_{A}^{+}} \eta(\boldsymbol{a u})\left\{\{A \rightarrow B\}_{\boldsymbol{x}, \boldsymbol{w}^{\prime \prime \prime}}^{f, \boldsymbol{g}} \vdash\{C C\}_{z}^{\tilde{c}}\right.}\left(\mathbf{C}_{\eta}\right)
\end{aligned}
$$

Quantifiers. In the case of the quantifiers the free variables $\boldsymbol{m}$ are obviously relevant, so in these cases we will make these explicit:

$(\forall \mathrm{L})$. By the induction hypothesis we have closed terms $\boldsymbol{a}, \boldsymbol{b}$ in $\mathrm{W}$ such that

(IH) $\mathrm{W}(\boldsymbol{x}, \boldsymbol{w}), \boldsymbol{m}<^{\tau} \tilde{\boldsymbol{m}}, \forall \boldsymbol{y} \sqsubset_{\tau_{A}^{-}} \boldsymbol{a x w} \tilde{\boldsymbol{m}}\left\{\{A(t[\boldsymbol{m}])\}_{\boldsymbol{y}}^{\boldsymbol{x}} \vdash\{[B\}\}_{w}^{\boldsymbol{b x} \tilde{\boldsymbol{m}}}\right.$

Let $\tilde{t}[\boldsymbol{m}]$ be a term such that $\boldsymbol{m} \prec^{\rho} \tilde{\boldsymbol{m}} \vdash t[\boldsymbol{m}] \prec^{\tau} \tilde{t}[\tilde{\boldsymbol{m}}]$, using (A7). Note also that 
$\left(\mathbf{Q}_{1}\right)$ and $\left(\mathbf{Q}_{3}\right)$ imply $\forall \boldsymbol{y} \sqsubset \boldsymbol{a} \forall \boldsymbol{z}<\boldsymbol{b} A \vdash \forall \boldsymbol{z}<\boldsymbol{b} \forall \boldsymbol{y} \sqsubset \boldsymbol{a} A$. Then

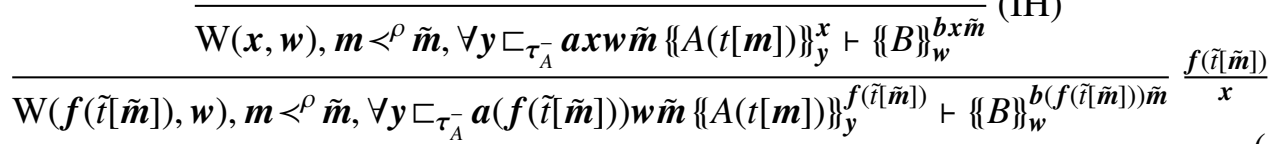

$$
\begin{aligned}
& \text { (IH) } \\
& \mathrm{W}(\boldsymbol{f}, \boldsymbol{w}), \boldsymbol{m}<^{\rho} \tilde{\boldsymbol{m}}, t[\boldsymbol{m}]<^{\tau} \tilde{t}[\tilde{\boldsymbol{m}}] \rightarrow \forall \boldsymbol{y} \sqsubset_{\tau_{A}^{-}} \boldsymbol{a}(\boldsymbol{f}(\tilde{t}[\tilde{\boldsymbol{m}}])) \boldsymbol{w} \tilde{\boldsymbol{m}}\left\{\{ A ( t [ \boldsymbol { m } ] ) \} _ { \boldsymbol { y } } ^ { f ( \tilde { t } [ \tilde { \boldsymbol { m } } ] ) } \vdash \left\{[B\}_{\boldsymbol{w}}^{\boldsymbol{b}(f(\tilde{f}[\tilde{\boldsymbol{m}}])) \tilde{\boldsymbol{m}}}(\rightarrow \mathrm{L})\right.\right.
\end{aligned}
$$

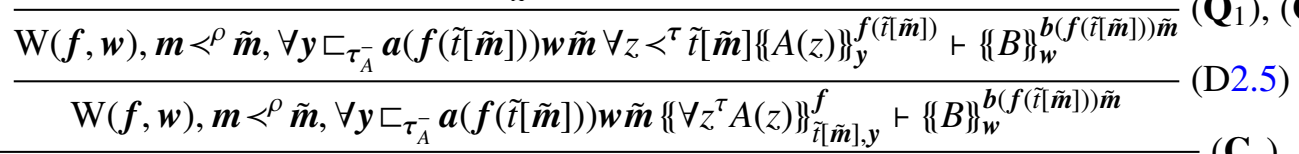

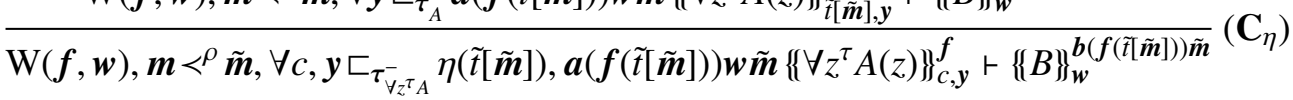

$(\forall \mathrm{R})$. This case is straightforward:

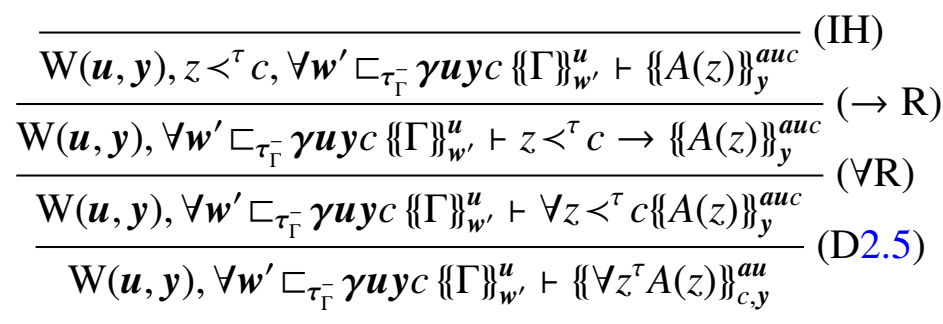

( $\exists \mathrm{L})$. By the induction hypothesis we have closed terms $\boldsymbol{a}, \boldsymbol{b}$ in $\mathrm{W}$ such that

(IH) $\mathrm{W}(\boldsymbol{x}, \boldsymbol{w}), z<^{\tau} c, \forall \boldsymbol{y} \sqsubset_{\tau_{A}^{-}} \boldsymbol{a x w} c\left\{\{A(z)\}_{\boldsymbol{y}}^{\boldsymbol{x}} \vdash\left\{\{B\}_{w}^{b x c}\right.\right.$

Then

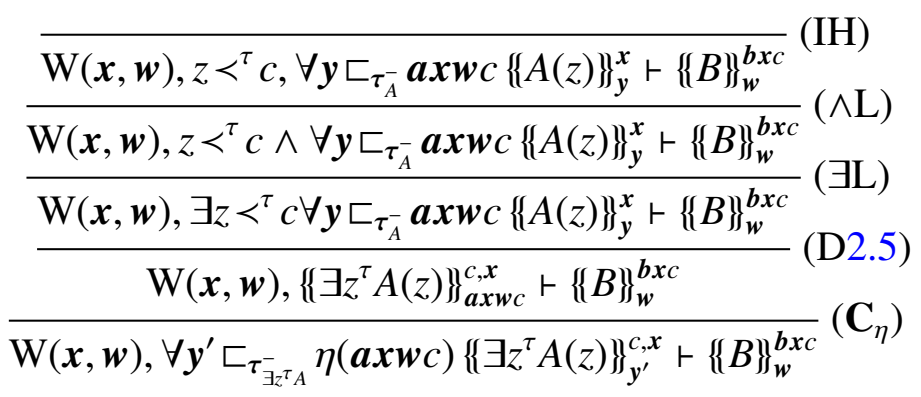


(ヨR). Let $\tilde{t}[\tilde{\boldsymbol{m}}]$ be the term such that $\boldsymbol{m} \prec^{\rho} \tilde{\boldsymbol{m}} \vdash t[\boldsymbol{m}]<^{\tau} \tilde{t}[\tilde{\boldsymbol{m}}]-$ see (A7). Then

$$
\begin{aligned}
& \overline{\mathrm{W}(\boldsymbol{u}, \boldsymbol{y}), \boldsymbol{m}<^{\rho} \tilde{\boldsymbol{m}}, \forall \boldsymbol{w}^{\prime} \sqsubset_{\tau_{\Gamma}^{-}} \gamma \boldsymbol{u} \tilde{\boldsymbol{m}} \boldsymbol{y}\left\{[ \Gamma \} _ { w ^ { \prime } } ^ { u } \vdash \left\{\{A(t[\boldsymbol{m}])\}_{\boldsymbol{y}}^{a u \tilde{m}}\right.\right.}(\mathrm{IH}) \\
& \overline{\mathrm{W}(\boldsymbol{u}, \boldsymbol{y}), \boldsymbol{m}<^{\rho} \tilde{\boldsymbol{m}}, \forall \boldsymbol{y}^{\prime} \sqsubset \boldsymbol{y} \forall \boldsymbol{w}^{\prime} \sqsubset_{\tau_{\Gamma}^{-}} \gamma \boldsymbol{u} \tilde{\boldsymbol{m}} \boldsymbol{y}^{\prime}\left\{[\Gamma\}_{\boldsymbol{w}^{\prime}}^{\boldsymbol{u}}+\forall \boldsymbol{y}^{\prime} \sqsubset \boldsymbol{y}\left\{\{A(t[\boldsymbol{m}])\}_{\boldsymbol{y}^{\prime}}^{a u \tilde{\boldsymbol{m}}}\right.\right.}\left(\mathbf{Q}_{1}\right),\left(\mathbf{Q}_{2}\right) \\
& \overline{\mathrm{W}(\boldsymbol{u}, \boldsymbol{y}), \boldsymbol{m} \prec^{\rho} \tilde{\boldsymbol{m}}, \forall \boldsymbol{y}^{\prime} \sqsubset \boldsymbol{y} \forall \boldsymbol{w}^{\prime} \sqsubset_{\tau_{\Gamma}^{-}} \gamma \boldsymbol{u} \tilde{\boldsymbol{m}} \boldsymbol{y}^{\prime}\left\{[\Gamma\}_{w^{\prime}}^{u} \vdash t[\boldsymbol{m}]<^{\tau} \tilde{t}[\tilde{\boldsymbol{m}}] \wedge \forall \boldsymbol{y}^{\prime} \sqsubset \boldsymbol{y}\left\{\{A(t[\boldsymbol{m}])\}_{\boldsymbol{y}^{\prime}}^{\boldsymbol{a} u \tilde{\boldsymbol{m}}}\right.\right.}(\wedge \mathrm{R}) \\
& \mathrm{W}(\boldsymbol{u}, \boldsymbol{y}), \boldsymbol{m}<^{\rho} \tilde{\boldsymbol{m}}, \forall \boldsymbol{w}^{\prime} \sqsubset_{\tau_{\Gamma}^{-}}(\gamma \boldsymbol{u} \tilde{\boldsymbol{m}}) \circ \boldsymbol{y}\left\{[ \Gamma \} _ { \boldsymbol { w } ^ { \prime } } ^ { \boldsymbol { u } } \vdash \left\{\{\exists z A(z)\}_{\boldsymbol{y}}^{\tilde{f} \tilde{\boldsymbol{m}}], a u \tilde{\boldsymbol{m}}}\right.\right.
\end{aligned}
$$

Weakening. By the induction hypothesis the premise of the weakening rule is witnessable, i.e. we have closed terms $\boldsymbol{\gamma}, \boldsymbol{b}$ in $\mathrm{W}$ such that

(IH) $\mathrm{W}(\boldsymbol{u}, \boldsymbol{w}), \forall \boldsymbol{w}^{\prime} \sqsubset_{\tau_{\Gamma}^{-}} \gamma \boldsymbol{u} \boldsymbol{w}\left\{[\Gamma\}_{w^{\prime}}^{u} \vdash\{\{B\}\}_{w}^{b u}\right.$

Let $\mathbf{0}$ be an arbitrary closed terms of the appropriate type. It follows that the terms $\lambda \boldsymbol{u}, \boldsymbol{x}, \boldsymbol{w} . \boldsymbol{\gamma} \boldsymbol{u} \boldsymbol{w}$ and $\mathbf{0}$ and $\lambda \boldsymbol{u}, \boldsymbol{x} . \boldsymbol{b} \boldsymbol{u}$ witness the conclusion of the weakening rule:

$$
\frac{\overline{\mathrm{W}(\boldsymbol{u}, \boldsymbol{w}), \forall \boldsymbol{w}^{\prime} \sqsubset_{\tau_{\Gamma}^{-}} \gamma \boldsymbol{u w}\left\{\{\Gamma\}_{w^{\prime}}^{u} \vdash\{\{B\}\}_{w}^{b u}\right.}(\mathrm{IH})}{\mathrm{W}(\boldsymbol{u}, \boldsymbol{w}, \boldsymbol{x}), \forall \boldsymbol{w}^{\prime} \sqsubset_{\tau_{\Gamma}^{-}} \gamma \boldsymbol{u} \boldsymbol{w}\left\{\{\Gamma\}_{w^{\prime}}^{u}, \forall \boldsymbol{y} \sqsubset_{\tau_{A}^{-}} 0 u \boldsymbol{x} w\left\{\{A\}_{y}^{x} \vdash\{\{B\}\}_{w}^{b u}\right.\right.}(\mathrm{wkn})
$$

Contraction. By the induction hypothesis we have closed terms $\boldsymbol{\gamma}, \boldsymbol{a}_{0}, \boldsymbol{a}_{1}, \boldsymbol{b}$ such that

$\left(\mathrm{IH}_{(i)}\right) \vdash \mathrm{W}(\boldsymbol{\gamma})$ and $\vdash \mathrm{W}\left(\boldsymbol{a}_{i}\right)$, for $i \in\{0,1\}$, and $\vdash \mathrm{W}(\boldsymbol{b})$

$\left(\mathrm{IH}_{(i i)}\right) \mathrm{W}\left(\boldsymbol{x}_{0}, \boldsymbol{x}_{1}, \boldsymbol{w}\right), \forall \boldsymbol{y} \sqsubset_{\tau_{A}^{-}} a_{0} x_{0} x_{1} w\left\{\{A\}_{y}^{\boldsymbol{x}_{0}}, \forall \boldsymbol{y} \sqsubset_{\tau_{A}^{-}} a_{1} x_{0} x_{1} w\left\{\{A\}_{y}^{x_{1}} \vdash\left\{\{B\}_{w}^{b x_{0} x_{1}}\right.\right.\right.$

We have Let $\tilde{\boldsymbol{a}_{0}}:=\boldsymbol{a}_{0} \boldsymbol{x} \boldsymbol{x} \boldsymbol{w}$ and $\tilde{\boldsymbol{a}_{1}}:=\boldsymbol{a}_{1} \boldsymbol{x} \boldsymbol{x} \boldsymbol{w}$. Then

$$
\begin{aligned}
& \frac{\overline{\mathrm{W}\left(x_{0}, x_{1}, w\right), \forall y \sqsubset \tau_{\tau_{A}^{-}} a_{0} x_{0} x_{1} w\left\{\{A\}_{y}^{x_{0}}, \forall y \sqsubset_{\tau_{A}^{-}} a_{1} x_{0} x_{1} w\left\{\{A\}_{y}^{x_{1}} \vdash\{B\}_{w}^{b x_{0} x_{1}}\right.\right.}\left(\mathrm{IH}_{(i i)}\right)}{\mathrm{W}(x, w), \forall y \sqsubset_{\tau_{A}^{-}} \tilde{a_{0}}\{\{A\}\}_{y}^{x}, \forall y \sqsubset_{\tau_{A}^{-}} \tilde{a_{1}}\left\{\{ A \} _ { y } ^ { x } \vdash \left\{\{B\}_{w}^{b x x}\right.\right.}\left[\frac{x}{x_{0}}, \frac{x}{x_{1}}\right] \\
& \frac{\mathrm{W}(\boldsymbol{x}, \boldsymbol{w}), \forall \boldsymbol{y} \sqsubset_{\tau_{A}^{-}} \tilde{\boldsymbol{a}_{0}}\left\{\{A\}_{y}^{x}, \forall \boldsymbol{y} \sqsubset_{\tau_{A}^{-}} \tilde{\boldsymbol{a}_{1}}\left\{\{ A \} _ { y } ^ { x } \vdash \left\{\{B\}_{w}^{b x \boldsymbol{x}}\right.\right.\right.}{\mathrm{W}(\boldsymbol{x}, \boldsymbol{w}), \forall \boldsymbol{y} \sqsubset_{\tau_{A}^{-}} \tilde{\boldsymbol{a}_{0}}\left\{\{ A \} _ { y } ^ { x } \wedge \forall \boldsymbol { y } \sqsubset _ { \tau _ { A } ^ { - } } \tilde { \boldsymbol { a } _ { 1 } } \{ \{ A \} \} _ { y } ^ { x } \vdash \left\{\{B\}_{w}^{\boldsymbol{b x x}}\right.\right.}(\wedge \mathrm{L}) \\
& \mathrm{W(x,w, \tilde {a_{0 } } , \tilde { a _ { 1 } } ) , \forall \boldsymbol { y } \sqsubset _ { \tau _ { A } ^ { - } } \tilde { \boldsymbol { a } _ { 0 } } \sqcup \tilde { \boldsymbol { a } _ { 1 } } \{ \{ A \} _ { y } ^ { x } \vdash \{ B \} _ { w } ^ { b x \boldsymbol { x } }}\left(\mathrm{IH}_{(i)}\right) \\
& \mathrm{W}(\boldsymbol{x}, \boldsymbol{w}), \forall \boldsymbol{y} \sqsubset_{\tau_{A}^{-}} \tilde{\boldsymbol{a}_{0}} \sqcup \tilde{\boldsymbol{a}_{1}}\left\{\{ A \} _ { y } ^ { x } \vdash \left\{\{B\}_{w}^{b x \boldsymbol{x}}\right.\right.
\end{aligned}
$$

Induction. Let us now consider the induction rule

$$
\frac{\vdash A(0) \quad A(n) \vdash A(n+1)}{\vdash \forall n^{\mathbb{N}} A(n)}
$$

Let $\boldsymbol{s}, \boldsymbol{r}, \boldsymbol{t}$ in $\mathrm{W}$ be realisers for the premises: 
(i) $\mathrm{W}(\boldsymbol{y}) \vdash_{\mathcal{A}_{\mathrm{t}}}\left\{\{A(0)\}_{\boldsymbol{y}}^{\boldsymbol{s}}\right.$

(ii) $\mathrm{W}(\boldsymbol{x}, \boldsymbol{y}), \forall \boldsymbol{y}^{\prime} \sqsubset_{\tau_{A}^{-}} \boldsymbol{r a x y}\left\{\{A(n)\}_{\boldsymbol{y}^{\prime}}^{\boldsymbol{x}}, n \prec^{\mathbb{N}} a \vdash_{\mathcal{A}_{\mathbf{t}}}\left\{\{A(n+1)\}_{\boldsymbol{y}}^{\operatorname{tax}}\right.\right.$

Using assumption (A6) that $n \prec^{\mathbb{N}} v n$, we have

(iii) $\mathrm{W}(\boldsymbol{x}, \boldsymbol{y}), \forall \boldsymbol{y}^{\prime} \sqsubset_{\tau_{A}^{-}} \boldsymbol{r}(v n) \boldsymbol{x} \boldsymbol{y}\left\{\{A(n)\}_{\boldsymbol{y}^{\prime}}^{\boldsymbol{x}} \vdash_{\mathcal{A}_{\mathbf{t}}}\left\{\{A(n+1)\}_{\boldsymbol{y}}^{t(v n) \boldsymbol{x}}\right.\right.$

which, by $\left(\mathbf{Q}_{2}\right)$, implies

(iv) $\mathrm{W}(\boldsymbol{x}), \forall \boldsymbol{y}^{\mathrm{W}}\left\{\{A(n)\}_{\boldsymbol{y}}^{\boldsymbol{x}} \vdash_{\mathcal{A}_{\mathrm{t}}} \forall \boldsymbol{y}^{\mathrm{W}}\left\{\{A(n+1)\}_{\boldsymbol{y}}^{t(v n) \boldsymbol{x}}\right.\right.$

Let $\boldsymbol{f} n$ be defined by primitive recursion on $n$ as

$$
\boldsymbol{f} n= \begin{cases}\boldsymbol{s} & \text { if } n=0 \\ \boldsymbol{t}(v n)(\boldsymbol{f}(n-1)) & \text { if } n>0\end{cases}
$$

Since $\mathrm{W}(\boldsymbol{s}, \boldsymbol{t}, v n)$, it follows that $\forall n^{\mathbb{N}} \mathrm{W}(f n)$. From (i) and (iv), by induction on $n$ we have

(v) $\mathrm{W}(\boldsymbol{y}) \vdash_{\mathcal{A}_{\mathbf{t}}}\left\{\{A(n)\}_{\boldsymbol{y}}^{f n}\right.$

and, by Lemma 2.9, W(y), $n \prec^{\mathbb{N}} a \vdash_{\mathcal{A}_{\mathbf{t}}}\left\{\{A(n)\}_{\boldsymbol{y}}^{\mathrm{m}_{A}^{\mathbb{N}}(f)(a)}\right.$.

\section{Concrete Interpretations of WE-HA ${ }^{\omega}$}

Let us now consider several instances of the parametrised interpretation $\{[\cdot\}\}$. By the parametrised Soundness Theorem 2.12, in order to prove the soundness of the instantiation, it is enough to check that the choice of parameters is adequate for the formulas in the image of the interpretation, and that the non-logical axioms of WE-HA ${ }^{\omega}$ are witnessable in $\mathcal{A}_{\mathbf{t}}$. For simplicity, for all instantiations considered here we always take the target theory to be $\mathcal{A}_{\mathbf{t}}=\mathbf{N}-\mathbf{H} \mathbf{A}^{\omega}$ (equality available for all types).

We consider three groups of instantiations, depending on the choice of the parameter $x<^{\tau} a$, which we will take to be either

1. $x={ }_{\tau} a$ with $\operatorname{wt}(\tau)=\tau$ (equality),

2. $x \in_{\tau} a$ with $\operatorname{wt}(\tau)=\tau^{*}$ (set inclusion) or

3. $x \leq_{\tau}^{*} a$ with $\operatorname{wt}(\tau)=\tau$ (majorizability).

In each of these cases, and for the corresponding instances of $\mathrm{W}$ considered here, it should be straightforward to verify that assumptions (A5), (A6) and (A7) are 
satisfied (disjunction, induction and finite types). For instance, in the case of majorizability, when $\mathrm{W}_{\tau}(x)=x \leq_{\tau}^{*} x$, assumption (A6) becomes

$$
\forall n^{\mathbb{N}}\left(f n \leq_{\tau}^{*} f n\right), n \leq a, x \leq_{\tau}^{*} f n \vdash x \leq_{\tau}^{*} \mathrm{~m}_{P}^{\mathbb{N}}(f)(a)
$$

which is satisfied for $\mathrm{m}_{P}^{\mathbb{N}}(f)(a)=\max _{n \leq a} f n$; and (A7) is the standard result that says that all terms of Gödel's system $T$ are majorizable.

\subsection{Interpretations where $x<^{\tau} a: \equiv x={ }_{\tau} a$}

The instances where $x<^{\tau} a$ is chosen to be $x={ }_{\tau} a$, with $\operatorname{wt}(\tau)=\tau$ and $m_{\tau}^{\mathbb{B}}(f)(a)=m_{\tau}^{\mathbb{N}}(f)(a)=f(a)$, which we call precise interpretations, include the seminal interpretations such as Gödel's Dialectica interpretation, its Diller-Nahm variant, and Kreisel's modified realizability. In these cases the soundness of the interpretation is already known, so we will simply show in detail how the parameters are instantiated to obtain these interpretations, without duelling too much on their soundness.

Modified realizability interpretation. Consider the following instantiation of the parameters:

$$
\begin{array}{ccccccc}
x<^{\tau} a & \operatorname{wt}(\tau) & \mathrm{W}_{\tau}(x) & \forall x \sqsubset_{\tau} \varepsilon A & \operatorname{bt}(\tau) & \mathrm{m}_{\tau}^{\mathbb{B}} & \mathrm{m}_{\tau}^{\mathbb{N}} \\
\hline x={ }_{\tau} a & \tau & \text { true } & \forall \boldsymbol{x}^{\tau} A & \varepsilon & \text { if } & \lambda f . f
\end{array}
$$

where $\varepsilon$ denotes the empty tuple of terms or types.

Proposition 3.1 (Kreisel's modified realizability of WE-HA ${ }^{\omega}$ - pointwise presentation). With the parameters instantiated as above we have:

$$
\begin{aligned}
& \{\{s=t\} \quad \Leftrightarrow \quad s=t \\
& \left\{\{A \wedge B\}_{y, w}^{x, v} \Leftrightarrow\{A A\}_{y}^{x} \wedge\{\{B\}\}_{w}^{v}\right. \\
& \left\{\{ A \vee B \} ^ { b , \boldsymbol { x } , \boldsymbol { v } } \Leftrightarrow \left(b=\mathrm{T} \wedge \forall \boldsymbol{y}\left\{\{A\}_{y}^{\boldsymbol{x}}\right) \vee\left(b=\mathrm{F} \wedge \forall \boldsymbol{w}\{\{B\}\}_{w}^{v}\right)\right.\right. \\
& \left\{\{ A \rightarrow B \} _ { x , w } ^ { f } \Leftrightarrow \forall \boldsymbol { y } \left\{\{ A \} _ { y } ^ { x } \rightarrow \left\{\{B\}_{w}^{f x}\right.\right.\right. \\
& \left\{\left\{\exists z^{\tau} A\right\}\right\}^{c, \boldsymbol{x}} \Leftrightarrow \forall \boldsymbol{y}\left\{\{A[c / z]\}_{\boldsymbol{y}}^{\boldsymbol{x}}\right. \\
& \left\{\{ \forall z ^ { \tau } A \} _ { c , \boldsymbol { y } } ^ { f } \Leftrightarrow \left\{\{A[c / z]\}_{\boldsymbol{y}}^{f_{c}}\right.\right.
\end{aligned}
$$

so that $\forall \boldsymbol{y}\{\{A\}\}_{\boldsymbol{y}}^{x} \Leftrightarrow \boldsymbol{x} \mathrm{mr} A(c f .[26])$. 
Dialectica interpretation. Consider this instantiation of the parameters:

\begin{tabular}{ccccccc}
$x<^{\tau} a$ & $\operatorname{wt}(\tau)$ & $\mathrm{W}_{\tau}(x)$ & $\forall \boldsymbol{x} \sqsubset_{\tau} \boldsymbol{a} A$ & $\mathrm{bt}(\boldsymbol{\tau})$ & $\mathrm{m}_{\tau}^{\mathbb{B}}$ & $\mathrm{m}_{\tau}^{\mathbb{N}}$ \\
\hline$x={ }_{\tau} a$ & $\tau$ & true & $A[\boldsymbol{a} / \boldsymbol{x}]$ & $\boldsymbol{\tau}$ & if $_{\tau}$ & $\lambda f . f$
\end{tabular}

Proposition 3.2 (Gödel's Dialectica interpretation of WE-HA ${ }^{\omega}$ ). With the parameters instantiated as above we have:

$$
\begin{aligned}
& \{\{s=t\} \quad \Leftrightarrow \quad s=t \\
& \{\{A \wedge B\}\}_{y, w}^{x, v} \Leftrightarrow\left\{\{A\}_{y}^{x} \wedge\{\{B\}\}_{w}^{v}\right. \\
& \left\{\{A \vee B\}_{y, w}^{b, \boldsymbol{x}, \boldsymbol{v}} \Leftrightarrow\left(b=\mathrm{T} \wedge\{\{A\}\}_{\boldsymbol{y}}^{\boldsymbol{x}}\right) \vee\left(b=\mathrm{F} \wedge\{\{B\}\}_{w}^{v}\right)\right. \\
& \left\{\{ A \rightarrow B \} _ { x , w } ^ { f , g } \Leftrightarrow \left\{\{A\}_{g x w}^{x} \rightarrow\{\{B\}\}_{w}^{f x}\right.\right. \\
& \left\{\left\{\exists z^{\tau} A\right\}\right\}_{\boldsymbol{y}}^{c, x} \Leftrightarrow\left\{\{A[c / z]\}_{\boldsymbol{y}}^{x}\right. \\
& \left\{\left\{\forall z^{\tau} A\right\}\right\}_{y, b}^{f} \Leftrightarrow\left\{\{A[b / z]\}_{\boldsymbol{y}}^{f b}\right.
\end{aligned}
$$

so that $\left\{\{A\}_{\boldsymbol{y}}^{x} \Leftrightarrow A_{D}(\boldsymbol{x} ; \boldsymbol{y})\right.$.

Proof. We are using here the equivalences

$$
\exists x^{\tau}\left(x={ }_{\tau} t \wedge A(x)\right) \Leftrightarrow A(t) \text { and } \forall x^{\tau}\left(x={ }_{\tau} t \rightarrow A(x)\right) \Leftrightarrow A(t)
$$

which are valid in $\mathbf{N}-\mathbf{H} \mathbf{A}^{\omega}$, in order to remove equality on higher-types. In this way, since our source theory is $\mathbf{W E - H A}{ }^{\omega}$, we also have that for all formulas $A$ of $\mathbf{W E}-\mathbf{H A} \mathbf{A}^{\omega}$, the formula $\left\{\{A\}_{y}^{x}\right.$ will be decidable. This property is essential for satisfying condition $\left(\mathbf{C}_{\sqcup}\right)$, where $\boldsymbol{y}_{1} \sqcup \boldsymbol{y}_{2}$ is defined via a case distinction involving the formula $\left\{\{A\}_{y}^{x}\right.$. This is also the only place where we make use of the assumption that the choice of parameters only needs to be adequate for the formulas in the image of the interpretation.

Diller-Nahm interpretation. Consider this instantiation of the parameters:

$$
\begin{array}{ccccccc}
x<{ }^{\tau} a & \operatorname{wt}(\tau) & \mathrm{W}_{\tau}(x) & \forall \boldsymbol{x} \sqsubset_{\tau} \boldsymbol{a} A & \operatorname{bt}(\boldsymbol{\tau}) & \mathrm{m}_{\tau}^{\mathbb{B}} & \mathrm{m}_{\tau}^{\mathbb{N}} \\
\hline x={ }_{\tau} a & \tau & \text { true } & \forall \boldsymbol{x} \in_{\tau} \boldsymbol{a} A & \boldsymbol{\tau}^{*} & \text { if }_{\tau} & \lambda f . f
\end{array}
$$

Proposition 3.3. With the parameters instantiated as above we have:

$$
\begin{aligned}
& \{\{s=t\}\} \quad \Leftrightarrow \quad s=t \\
& \left\{\{ A \wedge B \} _ { y , w } ^ { x , v } \Leftrightarrow \left\{\{ A \} _ { y } ^ { x } \wedge \left\{\{B\}_{w}^{v}\right.\right.\right. \\
& \{\{A \vee B\}\}_{\boldsymbol{y}, \boldsymbol{w}}^{b, \boldsymbol{x}, \boldsymbol{v}} \Leftrightarrow \quad\left(b=\mathrm{T} \wedge \forall \boldsymbol{y}^{\prime} \in_{\tau_{A}^{-}} \boldsymbol{y}\{\{A\}\}_{\boldsymbol{y}^{\prime}}^{\boldsymbol{x}}\right) \vee\left(b=\mathrm{F} \wedge \forall \boldsymbol{w}^{\prime} \in_{\tau_{B}^{-}} \boldsymbol{w}\left\{\{B\}_{\boldsymbol{w}^{\prime}}^{\boldsymbol{v}}\right)\right. \\
& \{A A \rightarrow B\}_{x, w}^{f, g} \Leftrightarrow \forall \boldsymbol{y} \in_{\tau_{A}^{-}} \boldsymbol{g x w}\left\{\{ A \} _ { y } ^ { x } \rightarrow \left\{\{B\}_{w}^{f x}\right.\right. \\
& \left\{\{ \exists z ^ { \tau } A \} _ { \boldsymbol { y } } ^ { c , \boldsymbol { x } } \Leftrightarrow \forall \boldsymbol { y } ^ { \prime } \in _ { \tau _ { A } ^ { - } } \boldsymbol { y } \left\{\{A[c / z]\}_{\boldsymbol{y}^{\prime}}^{\boldsymbol{x}}\right.\right. \\
& \left\{\forall \forall z^{\tau} A\right\}_{c, y}^{f} \Leftrightarrow\{A[c / z]\}_{\boldsymbol{y}}^{f c}
\end{aligned}
$$


The treatment of disjunction and the existential quantifier in the instantiation above appears to diverge from the standard Diller-Nahm interpretation, but the following proposition shows that this is in fact an equivalent way of presenting the Diller-Nahm interpretation.

Proposition 3.4 (Correspondence with Diller-Nahm interpretation of WE-HA ${ }^{\omega}$ ). The interpretation $\left\{\{A\}_{y}^{x}\right.$ in Proposition 3.3 can be seen to correspond to $A_{\mathrm{DN}}(\boldsymbol{x} ; \boldsymbol{y})$, in the sense that for each $A$ there are terms $\boldsymbol{s}_{1}, \boldsymbol{t}_{1}$ and $\boldsymbol{s}_{2}, \boldsymbol{t}_{2}$ such that

(i) $\forall y^{\prime} \in s_{1} x y\left\{\{A\}_{y^{\prime}}^{x} \vdash A_{\mathrm{DN}}\left(\boldsymbol{t}_{1} \boldsymbol{x} ; \boldsymbol{y}\right)\right.$

(ii) $\forall y^{\prime} \in s_{2} x y A_{\mathrm{DN}}\left(x ; y^{\prime}\right) \vdash\left\{\{A\}_{y}^{t_{2} x}\right.$

Proof. By induction on $A$.

Existential quantifier $(i)$. Let $s_{1}, t_{1}$ be given by the induction hypothesis. Then:

$$
\begin{array}{cl} 
& \forall \boldsymbol{y}^{\prime} \in\left\{\boldsymbol{s}_{1} \boldsymbol{x} \boldsymbol{y}\right\}\left\{\left\{\exists z^{\tau} A\right\}_{\boldsymbol{y}^{\prime}}^{c, \boldsymbol{x}}\right. \\
\stackrel{\mathrm{P} 3.3}{\Leftrightarrow} & \forall \boldsymbol{y}^{\prime} \in\left\{\boldsymbol{s}_{1} \boldsymbol{x} \boldsymbol{y}\right\} \forall \boldsymbol{y}^{\prime \prime} \in \boldsymbol{y}^{\prime}\{\{A[c / z]\}\}_{\boldsymbol{y}^{\prime \prime}}^{\boldsymbol{x}} \\
\Rightarrow & \forall \boldsymbol{y}^{\prime \prime} \in \boldsymbol{s}_{1} \boldsymbol{x} \boldsymbol{y}\{\{A[c / z]\}\}_{\boldsymbol{y}^{\prime \prime}}^{\boldsymbol{x}} \\
\stackrel{\left(\mathrm{IH}_{(i)}\right)}{\Rightarrow} & (A[c / z])_{\mathrm{DN}}\left(\boldsymbol{t}_{1} \boldsymbol{x} ; \boldsymbol{y}\right) \\
\stackrel{\mathrm{DN} \text { def. }}{\equiv} & \left(\exists z^{\tau} A\right)_{\mathrm{DN}}\left(c, \boldsymbol{t}_{1} \boldsymbol{x} ; \boldsymbol{y}\right)
\end{array}
$$

Existential quantifier (ii). Let $\boldsymbol{s}_{2}, \boldsymbol{t}_{2}$ be given by the induction hypothesis. Then:

$$
\begin{aligned}
& \forall \boldsymbol{y}^{\prime \prime} \in \bigcup_{\boldsymbol{y}^{\prime} \in \boldsymbol{y}} \boldsymbol{s}_{2} \boldsymbol{x} \boldsymbol{y}^{\prime}\left(\exists z^{\tau} A\right)_{\mathrm{DN}}\left(c, \boldsymbol{x} ; \boldsymbol{y}^{\prime \prime}\right) \\
& \stackrel{\mathrm{DN} \text { def. }}{\equiv} \forall \boldsymbol{y}^{\prime \prime} \in \bigcup_{\boldsymbol{y}^{\prime} \in \boldsymbol{y}} \boldsymbol{s}_{2} \boldsymbol{x} \boldsymbol{y}^{\prime}\left(A[c / z]_{\mathrm{DN}}\left(\boldsymbol{x} ; \boldsymbol{y}^{\prime \prime}\right)\right) \\
& \Rightarrow \quad \forall \boldsymbol{y}^{\prime \prime} \in \cup_{\boldsymbol{y}^{\prime} \in \boldsymbol{y}} \boldsymbol{s}_{2} \boldsymbol{x} \boldsymbol{y}^{\prime} A[c / z]_{\mathrm{DN}}\left(\boldsymbol{x} ; \boldsymbol{y}^{\prime \prime}\right) \\
& \Rightarrow \quad \forall \boldsymbol{y}^{\prime} \in \boldsymbol{y} \forall \boldsymbol{y}^{\prime \prime} \in \boldsymbol{s}_{2} \boldsymbol{x} \boldsymbol{y}^{\prime} A[c / z]_{\mathrm{DN}}\left(\boldsymbol{x} ; \boldsymbol{y}^{\prime \prime}\right) \\
& \stackrel{\left(\mathrm{IH}_{(i i)}\right)}{\Rightarrow} \forall \boldsymbol{y}^{\prime} \in \boldsymbol{y}\{\{A[c / z]\}\}_{y^{\prime}}^{t_{2} x} \\
& \stackrel{\mathrm{P} 3.3}{\Leftrightarrow}\left\{\left\{\left(\exists z^{\tau} A\right)\right\}\right\}_{\boldsymbol{y}^{\prime \prime}}^{c, t_{2} x}
\end{aligned}
$$

Implication (i). Let $\boldsymbol{s}_{1}^{B}, \boldsymbol{t}_{1}^{B}$ and $\boldsymbol{s}_{2}^{A}, \boldsymbol{t}_{2}^{A}$ be given by the induction hypothesis. Then: 


$$
\begin{aligned}
& \forall \boldsymbol{x}^{\prime} \in\left\{\boldsymbol{t}_{2}^{A} \boldsymbol{x}\right\} \forall \boldsymbol{w}^{\prime} \in \boldsymbol{s}_{1}^{B}\left(\boldsymbol{f} \boldsymbol{x}^{\prime}\right) \boldsymbol{w}\left\{\{A \rightarrow B\}_{x^{\prime}, w^{\prime}}^{f, g}\right. \\
& \Rightarrow \quad \forall \boldsymbol{w}^{\prime} \in \boldsymbol{s}_{1}^{B}\left(\boldsymbol{f}\left(\boldsymbol{t}_{2}^{A} \boldsymbol{x}\right)\right) \boldsymbol{w}\{\{A \rightarrow B\}\}_{t_{2}^{A} \boldsymbol{x}, \boldsymbol{w}^{\prime}}^{f, g} \\
& \stackrel{\mathrm{P} 3.3}{\Leftrightarrow} \forall \boldsymbol{w}^{\prime} \in \boldsymbol{s}_{1}^{B}\left(\boldsymbol{f}\left(\boldsymbol{t}_{2}^{A} \boldsymbol{x}\right)\right) \boldsymbol{w}\left(\forall \boldsymbol{y} \in \boldsymbol{g}\left(\boldsymbol{t}_{2}^{A} \boldsymbol{x}\right) \boldsymbol{w}^{\prime}\{\{A\}\}_{\boldsymbol{y}}^{\boldsymbol{t}_{2}^{A} \boldsymbol{x}} \rightarrow\{\{B\}\}_{w^{\prime}}^{f\left(t_{2}^{A} x\right)}\right) \\
& \Rightarrow \quad \forall w^{\prime} \in s_{1}^{B}\left(f\left(t_{2}^{A} x\right)\right) w \forall y \in g\left(t_{2}^{A} x\right) w^{\prime}\{\{A\}\}_{y}^{t_{2}^{A} x} \\
& \rightarrow \forall w^{\prime} \in s_{1}^{B}\left(f\left(t_{2}^{A} x\right)\right) w\{\{B\}\}_{w^{\prime}}^{f\left(t_{2}^{A} x\right)} \\
& \stackrel{\left(\mathrm{IH}_{(i)}, \mathrm{IH}_{(i i)}\right)}{\Rightarrow} \forall \boldsymbol{w}^{\prime} \in \boldsymbol{s}_{1}^{B}\left(\boldsymbol{f}\left(\boldsymbol{t}_{2}^{A} \boldsymbol{x}\right)\right) \boldsymbol{w} \forall \boldsymbol{y} \in \boldsymbol{g}\left(\boldsymbol{t}_{2}^{A} \boldsymbol{x}\right) \boldsymbol{w}^{\prime} \forall \boldsymbol{y}^{\prime} \in \boldsymbol{s}_{2}^{A} \boldsymbol{x y} A_{\mathrm{DN}}\left(\boldsymbol{x} ; \boldsymbol{y}^{\prime}\right) \\
& \rightarrow B_{\mathrm{DN}}\left(\boldsymbol{t}_{1}^{B}\left(\boldsymbol{f}\left(\boldsymbol{t}_{2}^{A} \boldsymbol{x}\right)\right) ; \boldsymbol{w}\right) \\
& \Rightarrow \quad \forall y^{\prime} \in \bigcup_{w^{\prime} \in s_{1}^{B}\left(f\left(t_{2}^{A} x\right)\right) w} \bigcup_{y \in g\left(t_{2}^{A} x\right) w^{\prime}} s_{2}^{A} x y A_{\mathrm{DN}}\left(x ; y^{\prime}\right) \\
& \rightarrow B_{\mathrm{DN}}\left(\boldsymbol{t}_{1}^{B}\left(\boldsymbol{f}\left(\boldsymbol{t}_{2}^{A} \boldsymbol{x}\right)\right) ; \boldsymbol{w}\right) \\
& \stackrel{\mathrm{DN} \text { def. }}{\equiv} \quad(A \rightarrow B)_{\mathrm{DN}}\left(\boldsymbol{t}_{1}^{A \rightarrow B}[\boldsymbol{f}, \boldsymbol{g}] ; \boldsymbol{x}, \boldsymbol{w}\right)
\end{aligned}
$$

where $\boldsymbol{t}_{1}^{A \rightarrow B}[\boldsymbol{f}, \boldsymbol{g}] \equiv \lambda \boldsymbol{x}, \boldsymbol{w} \cdot \bigcup_{\boldsymbol{w}^{\prime} \in \boldsymbol{s}_{1}^{B}\left(f\left(t_{2}^{A} \boldsymbol{x}\right)\right) \boldsymbol{w}} \bigcup_{\boldsymbol{y} \in \boldsymbol{g}\left(\boldsymbol{t}_{2}^{A} \boldsymbol{x}\right) \boldsymbol{w}^{\prime}} \boldsymbol{s}_{2}^{A} \boldsymbol{x} \boldsymbol{y}, \lambda \boldsymbol{x} . \boldsymbol{t}_{1}^{B}\left(\boldsymbol{f}\left(\boldsymbol{t}_{2}^{A} \boldsymbol{x}\right)\right)$.

Implication (ii). Let $\boldsymbol{s}_{1}^{A}, \boldsymbol{t}_{1}^{A}$ and $\boldsymbol{s}_{2}^{B}, \boldsymbol{t}_{2}^{B}$ be given by the induction hypothesis. Then:

$$
\begin{aligned}
& \forall \boldsymbol{x}^{\prime} \in\left\{\boldsymbol{t}_{1}^{A} \boldsymbol{x}\right\} \forall \boldsymbol{w}^{\prime} \in \boldsymbol{s}_{2}^{B}\left(\boldsymbol{f} \boldsymbol{x}^{\prime}\right) \boldsymbol{w}(A \rightarrow B)_{\mathrm{DN}}\left(\boldsymbol{f}, \boldsymbol{g} ; \boldsymbol{x}^{\prime}, \boldsymbol{w}^{\prime}\right) \\
& \Rightarrow \quad \forall \boldsymbol{w}^{\prime} \in \boldsymbol{s}_{2}^{B}\left(\boldsymbol{f}\left(\boldsymbol{t}_{1}^{A} \boldsymbol{x}\right)\right) \boldsymbol{w}(A \rightarrow B)_{\mathrm{DN}}\left(\boldsymbol{f}, \boldsymbol{g} ; \boldsymbol{t}_{1}^{A} \boldsymbol{x}, \boldsymbol{w}^{\prime}\right) \\
& \stackrel{\mathrm{DN} \text { def. }}{\equiv} \quad \forall \boldsymbol{w}^{\prime} \in \boldsymbol{s}_{2}^{B}\left(\boldsymbol{f}\left(\boldsymbol{t}_{1}^{A} \boldsymbol{x}\right)\right) \boldsymbol{w}\left(\forall \boldsymbol{y} \in \boldsymbol{g}\left(\boldsymbol{t}_{1}^{A} \boldsymbol{x}\right) \boldsymbol{w}^{\prime} A_{\mathrm{DN}}\left(\boldsymbol{t}_{1}^{A} \boldsymbol{x} ; \boldsymbol{y}\right)\right. \\
& \left.\rightarrow B_{\mathrm{DN}}\left(\boldsymbol{f}\left(\boldsymbol{t}_{1}^{A} \boldsymbol{x}\right) ; \boldsymbol{w}^{\prime}\right)\right) \\
& \Rightarrow \quad \forall \boldsymbol{w}^{\prime} \in \boldsymbol{s}_{2}^{B}\left(\boldsymbol{f}\left(\boldsymbol{t}_{1}^{A} \boldsymbol{x}\right)\right) \boldsymbol{w} \forall \boldsymbol{y} \in \boldsymbol{g}\left(\boldsymbol{t}_{1}^{A} \boldsymbol{x}\right) \boldsymbol{w}^{\prime} A_{\mathrm{DN}}\left(\boldsymbol{t}_{1}^{A} \boldsymbol{x} ; \boldsymbol{y}\right) \\
& \rightarrow \forall \boldsymbol{w}^{\prime} \in \boldsymbol{s}_{2}^{B}\left(\boldsymbol{f}\left(\boldsymbol{t}_{1}^{A} \boldsymbol{x}\right)\right) \boldsymbol{w} B_{\mathrm{DN}}\left(\boldsymbol{f}\left(\boldsymbol{t}_{1}^{A} \boldsymbol{x}\right) ; \boldsymbol{w}^{\prime}\right) \\
& \stackrel{\left(\mathrm{IH}_{(i)}, \mathrm{IH}_{(i i)}\right)}{\Rightarrow} \forall \boldsymbol{w}^{\prime} \in \boldsymbol{s}_{2}^{B}\left(\boldsymbol{f}\left(\boldsymbol{t}_{1}^{A} \boldsymbol{x}\right)\right) \boldsymbol{w} \forall \boldsymbol{y} \in \boldsymbol{g}\left(\boldsymbol{t}_{1}^{A} \boldsymbol{x}\right) \boldsymbol{w}^{\prime} \forall \boldsymbol{y}^{\prime} \in \boldsymbol{s}_{1}^{A} \boldsymbol{x y}\left\{\{A\}_{\boldsymbol{y}^{\prime}}^{\boldsymbol{x}}\right. \\
& \rightarrow\{\{B\}\}_{w}^{t_{2}^{B}\left(f\left(t_{1}^{A} x\right)\right)} \\
& \Rightarrow \quad \forall y^{\prime} \in \bigcup_{w^{\prime} \in s_{2}^{B}\left(f\left(t_{1}^{A} x\right)\right) w} \bigcup_{y \in g\left(t_{1}^{A} x\right) w^{\prime}} s_{1}^{A} x y\left\{\{A\}_{y^{\prime}}^{x} \rightarrow\{\{B\}\}_{w}^{t_{2}^{B}\left(f\left(t_{1}^{A} x\right)\right)}\right. \\
& \stackrel{\mathrm{P} 3.3}{\Leftrightarrow} \quad\{A \rightarrow B\}_{x, w}^{\lambda x, w . \cup_{w^{\prime} \in S_{2}^{B}\left(f\left(t_{1}^{A} x\right)\right) w} \cup_{y \in g\left(t_{1}^{A} x\right) w^{\prime}} s_{1}^{A} x y, \lambda x \cdot t_{2}^{B}\left(f\left(t_{1}^{A} x\right)\right)}
\end{aligned}
$$

Remark 3.5 (Stein's family of interpretations). In [28] Stein describes a family of interpretations parametrised by a number $n$. The idea is that when $\rho$ is a type of type level $\geq n$ we treat the contraction in a way similar to the Diller-Nahm interpretation and so $\operatorname{bt}(\rho)=\rho^{*}$ and $\forall x \sqsubset_{\rho} a A: \equiv \forall x \in_{\rho}$ range $(a) A$, where a is a function from the pure type $(n-1)$. But when $\rho$ is a type of type level $<n$ we treat it as modified realizability and therefore bt $(\rho)=\varepsilon$ and $\forall x \sqsubset_{\rho} \varepsilon A: \equiv \forall x^{\rho} A$. 
Although we could consider combinations of this with the various interpretations of quantifiers, we will leave this for future work.

Remark 3.6 (Diller-Nahm with majorizability). One could also consider the following choice of parameters

$$
\begin{array}{ccccccc}
x<^{\tau} a & \operatorname{wt}(\tau) & \mathrm{W}_{\tau}(x) & \forall \boldsymbol{x} \sqsubset_{\tau} \boldsymbol{a} A & \mathrm{bt}(\boldsymbol{\tau}) & \mathrm{m}_{\tau}^{\mathbb{B}} & \mathrm{m}_{\tau}^{\mathbb{N}} \\
\hline x={ }_{\tau} a & \tau & \text { true } & \forall \boldsymbol{x} \leq_{\tau}^{*} \boldsymbol{a} A & \boldsymbol{\tau} & \text { if }_{\tau} & \lambda f . f
\end{array}
$$

which corresponds to a version of the Diller-Nahm interpretation where set inclusion is replaced by majorizability. Unfortunately this does not seem to lead to a sound interpretation, and indeed we cannot satisfy condition $\left(\mathbf{C}_{\eta}\right)$, as there is no term $\eta$ which satisfies:

$$
\forall \boldsymbol{y} \leq_{\tau}^{*} \eta(z) A[\boldsymbol{y}]+A[z]
$$

in the target theory $\mathbf{N}-\mathbf{H} \mathbf{A}^{\omega}$ for an arbitrary $\boldsymbol{z}$, since this would imply $\boldsymbol{z} \leq_{\tau}^{*} \eta(\boldsymbol{z})$. One could then try to take $\left\{\mathrm{W}_{\tau}(x)\right\}_{\tau \in \mathcal{T}}$ to be " $x$ is monotone of type $\tau$ ", i.e. $\mathrm{W}_{\tau}(x)=$ $x \leq_{\tau}^{*} x$, but then assumption (A3) fails, since we no longer have $x<^{\tau} a \vdash \mathrm{W}_{\mathrm{wt}(\tau)}(a)$.

\subsection{Interpretations where $x<^{\tau} a: \equiv x \leq_{\tau}^{*} a$}

The instances where $x<^{\tau} a$ is chosen to be $x \leq_{\tau}^{*} a$, with wt $(\tau)=\tau$, which we call bounded interpretations, include the bounded functional interpretation [12], and the bounded modified realizability [11]. In this case we will also discuss a new interpretation: the bounded Diller-Nahm interpretation. In the following we will write $\tilde{\forall} x^{\tau} A$ as an abbreviation for $\forall x^{\tau}\left(x \leq_{\tau}^{*} x \rightarrow A\right)$.

Bounded modified realizability. Consider this instantiation of the parameters:

$$
\begin{array}{ccccccc}
x<^{\tau} a & \operatorname{wt}(\tau) & \mathrm{W}_{\tau}(x) & \forall \boldsymbol{x} \sqsubset_{\tau} \varepsilon A & \operatorname{bt}(\tau) & \mathrm{m}_{\tau}^{\mathbb{B}}(b, x, y) & \mathrm{m}_{\tau}^{\mathbb{N}} \\
\hline x \leq_{\tau}^{*} a & \tau & x \leq_{\tau}^{*} x & \tilde{\forall} \boldsymbol{x}^{\tau} A & \varepsilon & \max _{\tau}(x, y) & \lambda f . f
\end{array}
$$

Definition 3.7. Two functional interpretations of WE-HA ${ }^{\omega}$ into $\mathcal{A}_{\mathbf{t}}$ are said to be equivalent if they have the same set of witnessable sequents.

Proposition 3.8 (Bounded modified realizability, [11]). With the parameters instantiated as above we have:

$$
\begin{array}{ll}
\{\{s=t\}\} & \Leftrightarrow s=t \\
\left\{\{A \wedge B\}_{y, w}^{x, v}\right. & \Leftrightarrow \quad\{A\}_{y}^{x} \wedge\left\{\{B\}_{w}^{v}\right. \\
\left\{\{A \vee B\}^{b, x, v}\right. & \Leftrightarrow \exists z \leq^{*} b\left(\left(z=\mathrm{T} \wedge \tilde{\forall} \boldsymbol{y}\left\{\{A\}_{y}^{x}\right) \vee\left(z=\mathrm{F} \wedge \tilde{\forall} \boldsymbol{w}\{\{B\}\}_{w}^{v}\right)\right)\right. \\
\left\{\{A \rightarrow B\}_{x, \boldsymbol{w}}^{f}\right. & \Leftrightarrow \tilde{\forall} \boldsymbol{y}^{\tau_{A}^{-}}\left\{\{A\}_{\boldsymbol{y}}^{x} \rightarrow\{\{B\}\}_{w}^{f x}\right. \\
\left\{\left\{\exists z^{\tau} A\right\}\right\}^{c, \boldsymbol{x}} & \Leftrightarrow \exists z^{\tau} \leq_{\tau}^{*} c \tilde{\forall} \boldsymbol{y}\left\{\{A\}_{\boldsymbol{y}}^{x}\right. \\
\left\{\left\{\forall z^{\tau} A\right\}_{c, \boldsymbol{y}}^{f}\right. & \Leftrightarrow \forall z^{\tau} \leq_{\tau}^{*} c\left\{\{A\}_{\boldsymbol{y}}^{f c}\right.
\end{array}
$$


and $\{A A\}_{y}^{x}$ is equivalent to the bounded modified realizability.

Proof. Let us show that $\tilde{\exists} \boldsymbol{x} \tilde{\forall} \boldsymbol{y}\left\{\{\}_{y}^{x} \Leftrightarrow \tilde{\exists} \boldsymbol{x}(\boldsymbol{x}\right.$ bmr $A)$. We proof the two implications by induction on $A$. The only non-trivial case is disjunction. Assume first that we have a monotone terms $s, \boldsymbol{t}_{A}, \boldsymbol{t}_{B}$ such that

$$
\exists z \leq^{*} s\left(\left(z=\mathrm{T} \wedge \tilde{\forall} \boldsymbol{y}\{\{A\}\}_{y}^{t_{A}}\right) \vee\left(z=\mathrm{F} \wedge \tilde{\forall} \boldsymbol{w}\left\{[B\}_{w}^{t_{B}}\right)\right)\right.
$$

By induction hypothesis we have monotone terms $\boldsymbol{t}_{A}^{\prime}$ and $\boldsymbol{t}_{B}^{\prime}$ such that

$$
\exists z \leq^{*} s\left(\left(z=\mathrm{T} \wedge \boldsymbol{t}_{A}^{\prime} \mathrm{bmr} A\right) \vee\left(z=\mathrm{F} \wedge \boldsymbol{t}_{B}^{\prime} \mathrm{bmr} B\right)\right)
$$

which indeed implies $\left(\boldsymbol{t}_{A}^{\prime} \mathrm{bmr} A\right) \vee\left(\boldsymbol{t}_{B}^{\prime} \mathrm{bmr} B\right)$, and hence, $\boldsymbol{t}_{A}^{\prime}, \boldsymbol{t}_{B}^{\prime} \mathrm{bmr} A \vee B$.

On the other hand, assume we have monotone terms $\boldsymbol{t}_{A}^{\prime}$ and $\boldsymbol{t}_{B}^{\prime}$ such that

$$
\left(t_{A}^{\prime} \mathrm{bmr} A\right) \vee\left(t_{B}^{\prime} \mathrm{bmr} B\right) .
$$

Assuming $\mathrm{F} \leq \mathrm{T}$, we have

$$
\exists z \leq^{*} \mathrm{~T}\left(\left(z=\mathrm{T} \wedge \boldsymbol{t}_{A}^{\prime} \operatorname{bmr} A\right) \vee\left(z=\mathrm{F} \wedge \boldsymbol{t}_{B}^{\prime} \mathrm{bmr} B\right)\right)
$$

which by the induction hypothesis gives us monotone terms $\boldsymbol{t}_{A}$ and $\boldsymbol{t}_{B}$ such that

$$
\exists z \leq^{*} s\left(\left(z=\mathrm{T} \wedge \tilde{\forall} \boldsymbol{y}\left\{\{A\}_{y}^{t_{A}}\right) \vee\left(z=\mathrm{F} \wedge \tilde{\forall} \boldsymbol{w}\left\{[B\}_{w}^{t_{B}}\right)\right)\right.\right.
$$

and hence $\left\{\{A \vee B\}^{\mathrm{T}, t_{A}, t_{B}}\right.$.

Bounded functional interpretation. Consider this instantiation of the parameters:

$$
\begin{array}{ccccccc}
x<^{\tau} a & \operatorname{wt}(\tau) & \mathrm{W}_{\tau}(x) & \forall x \sqsubset_{\tau} \boldsymbol{a} A & \mathrm{bt}(\tau) & \mathrm{m}_{\tau}^{\mathbb{B}}(b, x, y) & \mathrm{m}_{\tau}^{\mathbb{N}} \\
\hline x \leq_{\tau}^{*} a & \tau & x \leq_{\tau}^{*} x & \tilde{\forall} \boldsymbol{x} \leq_{\tau}^{*} \boldsymbol{a} A & \boldsymbol{\tau} & \max _{\tau}(x, y) & \lambda f . f
\end{array}
$$

Proposition 3.9 (Bounded functional interpretation, [12]). With the parameters instantiated as above we have:

$$
\begin{aligned}
& \{\{s=t\}\} \quad \Leftrightarrow \quad s=t \\
& \left\{\{A \wedge B\}_{y, w}^{x, v} \Leftrightarrow\{A\}\right\}_{y}^{x} \wedge\left\{\{B\}_{w}^{v}\right. \\
& \left\{\{A \vee B\}_{y, w}^{b, \boldsymbol{x}, \boldsymbol{v}} \Leftrightarrow \exists z \leq^{*} b\left(\left(z=\mathrm{T} \wedge \tilde{\forall} \boldsymbol{y}^{\prime} \leq_{\tau_{A}^{*}}^{*} \boldsymbol{y}\left\{\{A\}_{\boldsymbol{y}^{\prime}}^{\boldsymbol{x}}\right) \vee\left(z=\mathrm{F} \wedge \tilde{\forall} \boldsymbol{w}^{\prime} \leq_{\tau_{B}^{-}}^{*} \boldsymbol{w}\left\{[B\}_{w^{\prime}}^{v}\right)\right)\right.\right.\right. \\
& \left\{\{A \rightarrow B\}_{x, w}^{f, g} \Leftrightarrow \tilde{\forall} \boldsymbol{y} \leq_{\tau_{A}^{-}}^{*} \boldsymbol{g x w}\left\{\{ A \} _ { y } ^ { x } \rightarrow \left\{\{B\}_{w}^{f x}\right.\right.\right. \\
& \left\{\exists z^{\tau} A\right\}_{y}^{c, \boldsymbol{x}} \Leftrightarrow \exists z \leq_{\tau}^{*} c \tilde{\forall} \boldsymbol{y}^{\prime} \leq_{\tau_{A}^{-}}^{*} \boldsymbol{y}\left\{\{A\}_{\boldsymbol{y}^{\prime}}^{\boldsymbol{x}}\right. \\
& \left\{\forall \forall z^{\tau} A\right\}_{c, y}^{f} \Leftrightarrow \forall z^{\tau} \leq_{\tau}^{*} c\{A A\}_{y}^{f_{c}^{A}}
\end{aligned}
$$

and $\left\{\{A\}_{y}^{x}\right.$ is equivalent to the bounded functional interpretation. 
Proof. Similar to Proposition 3.8.

Remark 3.10. In order to extend the source theory with bounded quantifiers, in this case one must add an "intensional" majorizability relation $x \unlhd y$, which satisfies

$$
f \unlhd g \quad \Rightarrow \quad \forall x, y(x \unlhd y \rightarrow(f x \unlhd g y) \wedge(f x \unlhd f y))
$$

with a rule-version of the other direction:

$$
\frac{\Gamma, x \unlhd y \vdash(f x \unlhd g y) \wedge(f x \unlhd f y)}{\Gamma \vdash f \unlhd g}
$$

Adding the other direction as an axiom would require us to produce a majorant for arbitrary x's and y's, which we do not have in the target theory $\mathbf{N}-\mathbf{H A}^{\omega}$.

Bounded Diller-Nahm interpretation. Let us consider now what we believe is a novel functional interpretation of $\mathbf{W E - H A}{ }^{\omega}$, where contraction is treated like in the Diller-Nahm interpretation (via finite sets), but the typing axioms are treated as in the bounded interpretations (via majorizability). As above, we are considering the source theory to be WE-HA ${ }^{\omega}$ and the target theory to be $\mathbf{N}-\mathbf{H A}{ }^{\omega}$, but consider the following instantiation of the parameters:

$$
\begin{array}{ccccccc}
x<^{\tau} a & \operatorname{wt}(\tau) & \mathrm{W}_{\tau}(x) & \forall \boldsymbol{x} \sqsubset_{\tau} \boldsymbol{a} A & \operatorname{bt}(\boldsymbol{\tau}) & \operatorname{m}_{\tau}^{\mathbb{B}}(b, x, y) & \mathrm{m}_{\tau}^{\mathbb{N}} \\
\hline x \leq_{\tau}^{*} a & \tau & x \leq_{\tau}^{*} x & \tilde{\forall} \boldsymbol{x} \in_{\tau} \boldsymbol{a} A & \boldsymbol{\tau}^{*} & \max _{\tau}(x, y) & \lambda f . f
\end{array}
$$

With these parameters the $\{\{\cdot\}\}$-interpretation becomes:

$$
\begin{aligned}
& \{\{s=t\} \quad \equiv s=t \\
& \left\{\{ A \wedge B \} _ { y , w } ^ { x , v } \equiv \left\{\{A\}_{y}^{x} \wedge\{\{B\}\}_{w}^{v}\right.\right. \\
& \left\{\{A \vee B\}_{\boldsymbol{y}, \boldsymbol{w}}^{b, \boldsymbol{x}, \boldsymbol{v}} \equiv \exists z \leq^{*} b\left(\left(z=\mathrm{T} \wedge \tilde{\forall} \boldsymbol{y}^{\prime} \in_{\tau_{A}^{-}} \boldsymbol{y}\left\{\{A\}_{\boldsymbol{y}^{\prime}}^{\boldsymbol{x}}\right) \vee\left(z=\mathrm{F} \wedge \tilde{\forall} \boldsymbol{w}^{\prime} \in \epsilon_{\tau_{B}^{-}} \boldsymbol{w}\left\{\{B\}_{\boldsymbol{w}^{\prime}}^{\boldsymbol{v}}\right)\right)\right.\right.\right.
\end{aligned}
$$

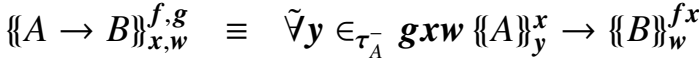

$$
\begin{aligned}
& \left\{\left\{\exists z^{\tau} A\right\}\right\}_{\boldsymbol{y}}^{c, \boldsymbol{x}} \equiv \exists z \leq_{\tau}^{*} c \tilde{\forall} \boldsymbol{y}^{\prime} \in \in_{\tau_{A}^{-}} \boldsymbol{y}\left\{\{A\}_{\boldsymbol{y}^{\prime}}^{\boldsymbol{x}}\right. \\
& \left\{\left\{\forall z^{\tau} A\right\}_{b, \boldsymbol{y}}^{f} \equiv \forall z \leq_{\tau}^{*} b\left\{\{A\}_{\boldsymbol{y}}^{f b}\right.\right.
\end{aligned}
$$

Proposition 3.11 (Bounded Diller-Nahm interpretation). The derived functional interpretation above is a sound interpretation of $\mathbf{W E}-\mathbf{H} \mathbf{A}^{\omega}$.

Proof. In order to prove the soundness for the interpretation it is enough to show that this choice of parameters is adequate for the formulas $\{A A\}_{y}^{x}$, for all $A$ in WE-HA $^{\omega}$. Since $\mathrm{W}_{\tau}(x)$ is the assumption that $x$ is self-majorizing (i.e. monotone $)$, conditions $\left(\mathbf{W}_{\mathrm{S}}\right),\left(\mathbf{W}_{\mathrm{K}}\right),\left(\mathbf{W}_{\mathrm{Ap}}\right)$ easily follow. $\left(\mathbf{Q}_{1}\right)$ and $\left(\mathbf{Q}_{2}\right)$ are also straightforward. The conditions for validating "contraction" $\left(\mathbf{C}_{\eta}\right),\left(\mathbf{C}_{\sqcup}\right),\left(\mathbf{C}_{\circ}\right)$ hold by taking $\eta(x)=\{x\}$ and $\boldsymbol{y}_{1} \sqcup \boldsymbol{y}_{2}=\boldsymbol{y}_{1} \cup \boldsymbol{y}_{2}$ and $\boldsymbol{f} \circ \boldsymbol{z}=\cup_{\boldsymbol{x} \in z} \boldsymbol{f} \boldsymbol{x}$ as indeed we have: 
$\left(\mathbf{C}_{\eta}\right)\left(\boldsymbol{z} \leq^{*} \boldsymbol{z}\right), \tilde{\forall} \boldsymbol{y} \in\{\boldsymbol{z}\} A[\boldsymbol{y}] \vdash_{\mathcal{A}_{\mathbf{t}}} A[\boldsymbol{z}]$

$\left(\mathbf{C}_{\sqcup}\right) \tilde{\forall} \boldsymbol{y} \in \boldsymbol{y}_{1} \cup \boldsymbol{y}_{2} A[\boldsymbol{y}] \vdash \mathcal{A}_{\mathbf{t}} \tilde{\forall} \boldsymbol{y} \in \boldsymbol{y}_{1} A[\boldsymbol{y}] \wedge \tilde{\forall} \boldsymbol{y} \in \boldsymbol{y}_{2} A[\boldsymbol{y}]$

$\left(\mathbf{C}_{\circ}\right) \tilde{\forall} \boldsymbol{y} \in \cup_{\boldsymbol{x} \in z} \boldsymbol{f} \boldsymbol{x} A[\boldsymbol{y}] \vdash_{\mathcal{A}_{\mathbf{t}}} \tilde{\forall} \boldsymbol{x} \in \boldsymbol{z} \tilde{\forall} \boldsymbol{y} \in \boldsymbol{f} \boldsymbol{x} A[\boldsymbol{y}]$

That concludes the proof that the choice of parameters is an adequate choice for the formulas $\left\{\{A\}_{y}^{x}\right.$, for all $A$ in $\mathbf{W E}-\mathbf{H} \mathbf{A}^{\omega}$.

Remark 3.12. It could turn out, however, that this "Bounded Diller-Nahm interpretation" is actually equivalent (in the sense of having the same characterising principles) as the Diller-Nahm interpretation or the bounded functional interpretation. Even though this is still open, we suspect this will not be the case, since being a member of a finite set is strictly stronger than being majorized by some element. More precisely, from $x \in_{\tau}$ a we indeed have $x \leq{ }^{*} \max a$. But from the assumption $x \leq_{\tau}^{*}$ a we cannot in general find a finite set $\tilde{a}$ (depending only on a) such that $x \in \tilde{a}$. This should be settled once we have investigated the characterising principles of this new interpretation, which we plan to do in a follow up paper.

\subsection{Interpretations where $x<^{\tau} a: \equiv x \in_{\tau} a$}

The instances where $x<^{\tau} a$ is chosen to be $x \in_{\tau} a$, with $\operatorname{wt}(\tau)=\tau^{*}$ and $\mathrm{m}_{P}^{\mathbb{N}}(f)(a)=\bigcup\{f z: z \in a\}$, which we call Herbrand interpretations, give some new interpretations for $\mathbf{W E}-\mathbf{H A}{ }^{\omega}{ }^{\omega}$ which are related with recently developed functional interpretations for nonstandard arithmetic [3]. In fact, to obtain the latter interpretations one needs to consider two types of predicate symbols, as explained in Section 4.

Herbrand realizability (for WE-HA ${ }^{\omega}$ ). Consider the following instantiation of the parameters:

$$
\begin{array}{ccccccc}
x<^{\tau} a & \operatorname{wt}(\tau) & \mathrm{W}_{\tau}(x) & \forall \boldsymbol{x} \sqsubset_{\tau} \varepsilon A & \operatorname{bt}(\tau) & \mathrm{m}_{\tau}^{\mathbb{B}}(b, x, y) & \mathrm{m}_{\tau}^{\mathbb{N}}(f)(a) \\
\hline x \in_{\tau} a & \tau^{*} & \text { true } & \forall \boldsymbol{x}^{\tau} A & \varepsilon & x \cup y & \bigcup_{z \in a} f z
\end{array}
$$

Proposition 3.13 (Herbrand realizability). With the parameters instantiated as above we have:

$$
\begin{array}{ll}
\{\{s=t\}\} & \Leftrightarrow s=t \\
\left\{\{A \wedge B\}_{y, w}^{x, v}\right. & \Leftrightarrow \quad\{A A\}_{y}^{x} \wedge\left\{\{B\}_{w}^{v}\right. \\
\{\{A \vee B\}\}^{b, x, v} & \Leftrightarrow \exists z \in b\left(\left(b=\mathrm{T} \wedge \forall \boldsymbol{y}\{\{A\}\}_{\boldsymbol{y}}^{x}\right) \vee\left(b=\mathrm{F} \wedge \forall \boldsymbol{w}\left\{\{B\}_{w}^{v}\right)\right)\right. \\
\left\{\{A \rightarrow B\}_{x, \boldsymbol{w}}^{f}\right. & \Leftrightarrow \forall \boldsymbol{y}^{\tau_{A}^{-}}\left\{\{A\}_{\boldsymbol{y}}^{x} \rightarrow\{\{B\}\}_{w}^{f x}\right. \\
\left\{\left\{\exists z^{\tau} A\right\}^{x, c}\right. & \Leftrightarrow \exists z \in_{\tau} c \forall \boldsymbol{y}\left\{\{A\}_{y}^{x}\right. \\
\left\{\left\{\forall z^{\tau} A\right\}_{c, \boldsymbol{y}}^{f}\right. & \Leftrightarrow \forall z \in_{\tau} c\{\{A\}\}_{y}^{f c}
\end{array}
$$


and $\left\{\{A\}_{y}^{x}\right.$ is equivalent to the Herbrand realizability of [3] (when that interpretation is restricted to the theory $\left.\mathbf{W E}-\mathbf{H A}{ }^{\omega}\right)$.

Proof. The Herbrand realizability is presented in [3] as an interpretation of the "nonstandard" theory E-HA $\mathbf{A}_{\mathrm{st}}^{\omega *}$. But, as discussed in [3, Prop. 2.7], E-HA $\mathbf{A}_{\mathrm{st}}^{\omega *}$ is a conservative extension of E-HA ${ }^{\omega}$, and hence also of $\mathbf{W E - H A}{ }^{\omega}$. It has been shown in [26] that this Herbrand realizability can be equivalently presented as:

$$
\begin{aligned}
& \varepsilon \mathrm{hr} s=t \quad \Leftrightarrow \quad s=t \\
& \boldsymbol{x}, \boldsymbol{v} \mathrm{hr} A \wedge B \quad \Leftrightarrow \quad \boldsymbol{x} \mathrm{hr} A \wedge \boldsymbol{v} \mathrm{hr} B \\
& \boldsymbol{x}, \boldsymbol{v} \operatorname{hr} A \vee B \quad \Leftrightarrow \quad \boldsymbol{x} \text { hr } A \vee v \operatorname{hr} B \\
& f \mathrm{hr} A \rightarrow B \Leftrightarrow \forall \boldsymbol{x}(\boldsymbol{x} \mathrm{hr} A \rightarrow \boldsymbol{f} \boldsymbol{x} \mathrm{hr} B) \\
& c, \boldsymbol{x} \mathrm{hr} \exists z^{\tau} A \Leftrightarrow \exists z \in_{\tau} c(\boldsymbol{x} \mathrm{hr} A) \\
& \boldsymbol{f} \mathrm{hr} \forall z^{\tau} A \quad \Leftrightarrow \quad \forall z \in_{\tau} c(\boldsymbol{f} c \mathrm{hr} A)
\end{aligned}
$$

It is straightforward to show (by induction on $A$ ) that $\exists \boldsymbol{x}(\boldsymbol{x}$ hr $A) \Leftrightarrow \exists \boldsymbol{x} \forall \boldsymbol{y}\left\{\{A\}_{y}{ }_{y}^{x}\right.$.

Herbrand Diller-Nahm interpretation. Consider the following instantiation of the parameters:

$$
\begin{array}{ccccccc}
x<^{\tau} a & \mathrm{wt}(\tau) & \mathrm{W}_{\tau}(x) & \forall \boldsymbol{x} \sqsubset_{\tau} \boldsymbol{a} A & \operatorname{bt}(\boldsymbol{\tau}) & \mathrm{m}_{\tau}^{\mathbb{B}}(b, x, y) & \mathrm{m}_{\tau}^{\mathbb{N}}(f)(a) \\
\hline x \in_{\tau} a & \tau^{*} & \text { true } & \forall \boldsymbol{x} \in_{\tau} \boldsymbol{a} A & \boldsymbol{\tau}^{*} & x \cup y & \bigcup_{z \in a} f z
\end{array}
$$

Proposition 3.14 (Herbrand Diller-Nahm interpretation). With the parameters instantiated as above we have:

$$
\begin{aligned}
& \{s=t\} \quad \Leftrightarrow s=t \\
& \left\{\{ A \wedge B \} _ { y , w } ^ { x , v } \Leftrightarrow \left\{\{A\}_{y}^{x} \wedge\{\{B\}\}_{w}^{v}\right.\right. \\
& \left\{\{ A \vee B \} _ { \boldsymbol { y } , \boldsymbol { w } } ^ { b , \boldsymbol { x } , \boldsymbol { v } } \Leftrightarrow \exists z \in b \left(\left(z=\mathrm{T} \wedge \forall \boldsymbol{y}^{\prime} \in_{\tau_{A}^{-}} \boldsymbol{y}\left\{\{A\}_{\boldsymbol{y}^{\prime}}^{\boldsymbol{x}}\right) \vee\left(z=\mathrm{F} \wedge \forall \boldsymbol{w}^{\prime} \in_{\tau_{B}^{-}} \boldsymbol{w}\{\{B\}\}_{\boldsymbol{w}^{\prime}}^{\boldsymbol{v}}\right)\right)\right.\right. \\
& \left\{\{ A \rightarrow B \} _ { x , w } ^ { f , g } \Leftrightarrow \forall \boldsymbol { y } \in _ { \tau _ { A } ^ { - } } \boldsymbol { g x \boldsymbol { w } } \left\{\{A\}_{\boldsymbol{y}}^{\boldsymbol{x}} \rightarrow\{\{B\}\}_{\boldsymbol{w}}^{f x}\right.\right. \\
& \left\{\left\{\exists z^{\tau} A\right\}_{\boldsymbol{y}}^{\boldsymbol{x}} \quad \Leftrightarrow \exists z \in_{\tau} c \forall \boldsymbol{y}^{\prime} \in_{\tau_{A}^{-}} \boldsymbol{y}\{\{A\}\}_{\boldsymbol{y}^{\prime}}^{\boldsymbol{x}}\right. \\
& \left\{\forall \forall z^{\tau} A\right\}_{b, \boldsymbol{y}}^{x} \Leftrightarrow \forall z \in_{\tau} b\left\{\{A\}_{\boldsymbol{y}}^{x}\right.
\end{aligned}
$$

and this is a sound interpretation of WE-HA ${ }^{\omega}$.

The Herbrand realizability for intuitionistic logic given by Proposition 3.13 and the Herbrand Diller-Nahm interpretation given by Proposition 3.14 are in a 
sense "rediscovered" interpretations. In fact, these interpretations are closely related with the interpretations given in [3] for WE-HA $\mathbf{A s t}_{\mathrm{st}}^{\omega}$. They are also closely connected with the interpretation for "pure logic" considered by Gilda Ferreira and Fernando Ferreira in the paper [9]. Moreover, Fernando Ferreira has a recent paper [8] where he considers essentially the Herbrand Diller-Nahm interpretation for WE-HA ${ }^{\omega}$ as well as an extension to second-order arithmetic.

Our parametrised interpretations allow us to consider also a Herbrand version of the bounded functional interpretation.

Herbrandized bfi. We conclude this list of instantiations with what we believe is another novel functional interpretation of $\mathbf{W E}-\mathbf{H} \mathbf{A}^{\omega}$, where contraction is treated like the Herbrandized interpretations, but the typing axioms are treated as in the bounded interpretations:

$$
\begin{array}{ccccccc}
x<^{\tau} a & \operatorname{wt}(\tau) & \mathrm{W}_{\tau}(x) & \forall \boldsymbol{x} \sqsubset_{\tau} \boldsymbol{a} A & \mathrm{bt}(\boldsymbol{\tau}) & \mathrm{m}_{\tau}^{\mathbb{B}}(b, x, y) & \mathrm{m}_{\tau}^{\mathbb{N}}(f)(a) \\
\hline x \in_{\tau} a & \tau^{*} & x \leq_{\tau}^{*} x & \tilde{\forall} \boldsymbol{x} \leq_{\tau}^{*} \boldsymbol{a} A & \boldsymbol{\tau} & x \cup y & \bigcup_{z \in a} f z
\end{array}
$$

Proposition 3.15 (Herbrandized bounded functional interpretation of WE-HA ${ }^{\omega}$ ). With the parameters instantiated as above we have:

$$
\begin{array}{ll}
\{\{s=t\}\} & \Leftrightarrow s=t \\
\left\{\{A \wedge B\}_{\boldsymbol{y}, \boldsymbol{w}}^{\boldsymbol{x}, \boldsymbol{w}}\right. & \Leftrightarrow\left\{\{A\}_{\boldsymbol{y}}^{x} \wedge\{\{B\}\}_{\boldsymbol{w}}^{v}\right. \\
\left\{\{A \vee B\}_{\boldsymbol{y}, \boldsymbol{w}}^{b, \boldsymbol{x}, \boldsymbol{v}}\right. & \Leftrightarrow \exists z \in b\left(\left(z=\mathrm{T} \wedge \tilde{\forall} \boldsymbol{y}^{\prime} \leq_{\tau_{A}^{-}} \boldsymbol{y}\{\{A\}\}_{\boldsymbol{y}^{\prime}}^{\boldsymbol{x}}\right) \vee\left(z=\mathrm{F} \wedge \tilde{\forall} \boldsymbol{w}^{\prime} \leq_{\tau_{B}^{-}}^{*} \boldsymbol{w}\left\{\{B\}_{w^{\prime}}^{v}\right)\right)\right. \\
\{\{A \rightarrow B\}\}_{\boldsymbol{x}, \boldsymbol{w}}^{f, \boldsymbol{g}} & \Leftrightarrow \tilde{\forall} \boldsymbol{y} \leq_{\tau_{A}^{-}}^{*} \boldsymbol{g} \boldsymbol{x} \boldsymbol{w}\{\{A\}\}_{\boldsymbol{y}}^{\boldsymbol{x}} \rightarrow\left\{\{B\}_{\boldsymbol{w}}^{f x}\right. \\
\left\{\left\{\exists z^{\tau} A\right\}_{y}^{c, \boldsymbol{x}}\right. & \Leftrightarrow \exists z \in_{\tau} c \tilde{\forall} \boldsymbol{y}^{\prime} \leq_{\tau_{A}^{-}}^{*} \boldsymbol{y}\left\{\{A\}_{\boldsymbol{y}^{\prime}}^{x}\right. \\
\left\{\left\{\forall z^{\tau} A\right\}_{c, \boldsymbol{y}}^{f}\right. & \Leftrightarrow \forall z \in_{\tau} c\left\{\{A\}_{\boldsymbol{y}}^{f c}\right.
\end{array}
$$

and this is a sound interpretation of $\mathbf{W E}-\mathbf{H A} \mathbf{A}^{\omega}$.

Proof. In order to show that this interpretation is sound, we should check that the choice of parameters is adequate - i.e. that (A1) - (A7) hold - which is quite straightforward and follows the same patterns as in the previous instances.

A remark similar to Remark 3.12 also applies here. We suspect that this is a new interpretation, but will only be certain once we have investigated its characterising principles. 
Remark 3.16 (A Herbrandized Dialectica). One might also consider an instantiation of the parameters as follows:

\begin{tabular}{ccccccc}
$x<^{\tau} a$ & $\mathrm{wt}(\tau)$ & $\mathrm{W}_{\tau}(x)$ & $\forall \boldsymbol{x} \sqsubset_{\tau} \boldsymbol{a} A$ & $\mathrm{bt}(\boldsymbol{\tau})$ & $\mathrm{m}_{\tau}^{\mathbb{B}}(b, x, y)$ & $\mathrm{m}_{\tau}^{\mathbb{N}}(f)(a)$ \\
\hline$x \in_{\tau} a$ & $\tau^{*}$ & true & $A[\boldsymbol{a} / \boldsymbol{x}]$ & $\boldsymbol{\tau}$ & $x \cup y$ & $\bigcup_{z \in a} f z$
\end{tabular}

which would correspond to a "Herbrandized" version of the Dialectica interpretation. In this case contraction is dealt with in a precise way, but quantifiers are approximated by finite sets. In WE-HA ${ }^{\omega}$, however, where definition by cases is available, it's easy to check that this would give rise to an interpretation which is equivalent to the original Dialectica, since the interpretation of the quantifiers

$$
\begin{aligned}
& \left\{\{ \exists z ^ { \tau } A \} _ { y } ^ { c , x } \Leftrightarrow \exists z \in _ { \tau } c \left\{\{A\}_{\boldsymbol{y}}^{x}\right.\right. \\
& \left\{\{ \forall z ^ { \tau } A \} _ { \boldsymbol { y } , c } ^ { f } \Leftrightarrow \forall z \in _ { \tau } c \left\{\{A\}_{y_{\boldsymbol{y}}}^{f_{c}}\right.\right.
\end{aligned}
$$

can be effectively replaced by precise witnesses

$$
\begin{array}{lll}
\left\{\exists \exists z^{\tau} A\right\}_{y}^{c, x} & \Leftrightarrow & \left\{\{A[c / z]\}_{y}^{x}\right. \\
\left\{\left\{\forall z^{\tau} A\right\}\right\}_{y, c}^{f} & \Leftrightarrow & \left\{\{A[c / z]\}_{y}^{f c}\right.
\end{array}
$$

\section{Final Remarks and Future Work}

We have described above a general framework for unifying several functional interpretations of WE-HA ${ }^{\omega}$, which we then used to discover new interpretations. The instantiations are summarised in Figure 1.

A notable family of functional interpretations that we are not covering in this paper is Kohlenbach's monotone functional interpretations (see [18, 19]). We focus here on the different ways a formula can be given a functional interpretation. The monotone functional interpretation in fact makes use of these same interpretations of formulas, but with a different interpretation of proofs. More precisely, given the interpretation of a formula $A$ as $\left\{\{A\}_{y}^{x}\right.$, we are focusing here on the soundness theorem that guarantees the existence of terms $t$ such that $\{\{A\}\}_{y}^{t}$ for provable $A$. In the monotone functional interpretation a different soundness proof is used, which, for provable $A$, guarantees the existence of terms $\tilde{\boldsymbol{t}}$ such that $\exists \boldsymbol{x} \leq^{*} \tilde{\boldsymbol{t}}\left\{\{A\}_{y}^{\boldsymbol{x}}\right.$, where $\leq^{*}$ is Bezem's strong majorizability relation. Hence, one could consider "monotone" soundness theorems for each of the interpretations discussed here, but we leave this to future work.

As shown in the previous section, the parametrised interpretations presented in this paper can be used as a way to discover new interpretations. The instances 


\begin{tabular}{c|c|c|c|c|c|c}
$\mathrm{wt}(\tau)$ & $x<^{\tau} a$ & $\mathrm{~m}_{\tau}^{\mathbb{B}}(b, x, y) / \mathrm{m}_{\tau}^{\mathbb{N}}(f, a)$ & $\mathrm{bt}(\tau)$ & $\forall \boldsymbol{x} \check{\tau}_{\tau} \boldsymbol{a} A$ & $\mathrm{~W}_{\tau}(x)$ & Interpretation \\
\hline$\tau$ & $x=_{\tau} a$ & if $_{\tau}(b, x, y) / f(a)$ & $\boldsymbol{\tau}$ & $A[\boldsymbol{a} / \boldsymbol{x}]$ & true & Dialectica \\
$\tau$ & $x=_{\tau} a$ & $\mathrm{if}_{\tau}(b, x, y) / f(a)$ & $\varepsilon$ & $\forall \boldsymbol{x}^{\tau} A$ & true & Modified realizability \\
$\tau$ & $x=_{\tau} a$ & $\mathrm{if}_{\tau}(b, x, y) / f(a)$ & $\boldsymbol{\tau}$ & $\forall \boldsymbol{x} \leq_{\tau}^{*} \boldsymbol{a} A$ & true $/ x \leq_{\tau}^{*} x$ & (combination not sound) \\
$\tau$ & $x=_{\tau} a$ & $\mathrm{if}_{\tau}(b, x, y) / f(a)$ & $\boldsymbol{\tau}^{*}$ & $\forall \boldsymbol{x} \in_{\tau} \boldsymbol{a} A$ & true & Diller-Nahm \\
\hline$\tau$ & $x \leq_{\tau}^{*} a$ & $\max _{\tau}(x, y) / f(a)$ & $\boldsymbol{\tau}$ & $A[\boldsymbol{a} / \boldsymbol{x}]$ & $x \leq_{\tau}^{*} x$ & (combination not sound) \\
$\tau$ & $x \leq_{\tau}^{*} a$ & $\max _{\tau}(x, y) / f(a)$ & $\varepsilon$ & $\tilde{\forall} \boldsymbol{x}^{\tau} A$ & $x \leq_{\tau}^{*} x$ & Bounded modified realizability \\
$\tau$ & $x \leq_{\tau}^{*} a$ & $\max _{\tau}(x, y) / f(a)$ & $\boldsymbol{\tau}$ & $\tilde{\forall} \boldsymbol{x} \leq_{\tau}^{*} \boldsymbol{a} A$ & $x \leq_{\tau}^{*} x$ & Bounded functional interpretation \\
$\tau$ & $x \leq_{\tau}^{*} a$ & $\max _{\tau}(x, y) / f(a)$ & $\boldsymbol{\tau}^{*}$ & $\tilde{\forall} \boldsymbol{x} \in_{\tau} \boldsymbol{a} A$ & $x \leq^{*} x$ & Bounded Diller-Nahm \\
\hline$\tau^{*}$ & $x \in_{\tau} a$ & $x \cup y / \bigcup_{z \in a} f z$ & $\boldsymbol{\tau}$ & $A[\boldsymbol{a} / \boldsymbol{x}]$ & true & Herbrand Dialectica ( Dialectica) \\
$\tau^{*}$ & $x \in_{\tau} a$ & $x \cup y / \bigcup_{z \in a} f z$ & $\varepsilon$ & $\forall \boldsymbol{x}^{\tau} A$ & true & Herbrand realizability (for IL) \\
$\tau^{*}$ & $x \in_{\tau} a$ & $x \cup y / \bigcup_{z \in a} f z$ & $\tau$ & $\tilde{\forall} \boldsymbol{x} \leq_{\tau}^{*} \boldsymbol{a} A$ & $x \leq_{\tau}^{*} x$ & Herbrandized bfi \\
$\tau^{*}$ & $x \in_{\tau} a$ & $x \cup y / \bigcup_{z \in a} f z$ & $\tau^{*}$ & $\forall \boldsymbol{x} \in_{\tau} \boldsymbol{a} A$ & true & Herbrand Diller-Nahm
\end{tabular}

Figure 1: Summary of instantiations (with the two novel interpretations in bold)

that we considered are by no means exhaustive. For instance, we think that the interpretations for nonstandard arithmetic from [3, 6, 10] should also fit in our framework. The idea is to consider not just different interpretations of the typed quantifiers $\forall x^{\tau} A$ and $\exists x^{\tau} A$, but also the typed standard quantification $\forall x^{\text {st }_{\tau}} A$ and $\exists x^{\text {st }} A$. Suitable choices for these should lead to the known interpretations of nonstandard arithmetic, but might also give rise to new interpretations for nonstandard arithmetic. This study, however, goes behind the scope of this paper.

Another question concerns variants with truth [15]. We think that it may be possible to obtain the existing interpretations with truth, and maybe to find new ones, using our parametrised interpretations. We also leave this to future work.

Usually, functional interpretations are accompanied by a characterisation theorem where one shows the equivalence between a formula and its interpretation. In order to show such equivalence one requires some principles - typically, a form of Choice and of Markov's principle are among such principles - which are called the characteristic principles of the interpretation. In the case of our parametrised interpretation we do not know if such a (parametric) theorem holds. We were able to define parametrised characteristic principles and obtain the result but only assuming that the characteristic principles are interpretable (by themselves). This does not solve the problem since it may happen that the theory with the principles may not be consistent. However, for each particular instantiation described in this paper the parametrised characteristic principles indeed correspond to the actual characteristic principles of the interpretation obtained with that instantia- 
tion. So, it seems that if the resulting theory is consistent, then the parametrised interpretation admits a characterisation theorem.

Finally, it is well-known that intuitionistic functional interpretations are related with classical ones by means of a negative translation. For example, as shown in [1, 29], Jean-Louis Krivine's negative translation is the correct tool to connect Gödel's Dialectica with Shoenfield's interpretation. Other factorisations were obtained in $[5,14,29,27]$. It is our impression that composing our intuitionistic parametrised interpretation with various negative translations would entail parametrised classical interpretations that allows one to obtain all the standard interpretations for classical logic, showing factorisations are a general feature among functional interpretations. We also leave this to a future study.

\section{References}

[1] Jeremy Avigad. A variant of the double-negation translation. Technical Report CMU-PHIL-179, Carnegie Mellon University, United States of America, August 2006.

[2] Jeremy Avigad and Solomon Feferman. Gödel's functional ("Dialectica") interpretation. In Handbook of proof theory, volume 137 of Stud. Logic Found. Math., pages 337-405. North-Holland, Amsterdam, 1998.

[3] Benno van den Berg, Eyvind Briseid, and Pavol Safarik. A functional interpretation for nonstandard arithmetic. Annals of Pure and Applied Logic, 163(12):1962-1994, December 2012.

[4] Justus Diller and Werner Nahm. Eine Variante zur Dialectica-Interpretation der Heyting-Arithmetik endlicher Typen. Arch. Math. Logik Grundlagenforsch., 16:49-66, 1974.

[5] Bruno Dinis and Jaime Gaspar. Factorisation of the classical nonstandard bounded functional interpretation. (technical report).

[6] Bruno Dinis and Jaime Gaspar. Intuitionistic nonstandard bounded modified realisability and functional interpretation. Ann. Pure Appl. Logic, 169(5):392-412, 2018.

[7] Bruno Dinis and Paulo Oliva. Parametrised functional interpretations. ArXiv 2005.05794, May 2020. 
[8] Fernando Ferreira. The FAN principle and weak König's lemma in herbrandized second-order arithmetic. Annals of Pure and Applied Logic, 171(9):102843, 2020.

[9] Fernando Ferreira and Gilda Ferreira. A herbrandized functional interpretation of classical first-order logic. Archive for Mathematical Logic, 56(5):523-539, Aug 2017.

[10] Fernando Ferreira and Jaime Gaspar. Nonstandardness and the bounded functional interpretation. Annals of Pure and Applied Logic, 166(6):701712, June 2015.

[11] Fernando Ferreira and Ana Nunes. Bounded modified realizability. The Journal of Symbolic Logic, 71(1):329-346, March 2006.

[12] Fernando Ferreira and Paulo Oliva. Bounded functional interpretation. Annals of Pure and Applied Logic, 135(1-3):73-112, September 2005.

[13] Gilda Ferreira and Paulo Oliva. Functional interpretations of intuitionistic linear logic. Log. Methods Comput. Sci., 7(1):1:9, 22, 2011.

[14] Jaime Gaspar. Factorization of the Shoenfield-like bounded functional interpretation. Notre Dame Journal of Formal Logic, 50(1):53-60, January 2009.

[15] Jaime Gaspar and Paulo Oliva. Proof interpretations with truth. MLQ Math. Log. Q., 56(6):591-610, 2010.

[16] Kurt Gödel. Über eine bisher noch nicht benützte Erweiterung des finiten Standpunktes. Dialectica, 12:280-287, 1958.

[17] Mircea-Dan Hernest and Paulo Oliva. Hybrid functional interpretations. In Logic and theory of algorithms, volume 5028 of Lecture Notes in Comput. Sci., pages 251-260. Springer, Berlin, 2008.

[18] Ulrich Kohlenbach. Analysing proofs in analysis. In Logic: from foundations to applications (Staffordshire, 1993), Oxford Sci. Publ., pages 225260. Oxford Univ. Press, New York, 1996.

[19] Ulrich Kohlenbach. Applied proof theory: proof interpretations and their use in mathematics. Springer Monographs in Mathematics. Springer-Verlag, Berlin, 2008. 
[20] Georg Kreisel. Interpretation of analysis by means of constructive functionals of finite types. In Constructivity in mathematics: Proceedings of the colloquium held at Amsterdam, 1957 (edited by A. Heyting), Studies in Logic and the Foundations of Mathematics, pages 101-128. North-Holland Publishing Co., Amsterdam, 1959.

[21] Paulo Oliva. Unifying functional interpretations. Notre Dame Journal of Formal Logic, 47(2):263-290, 2006.

[22] Paulo Oliva. Computational interpretations of classical linear logic. In Logic, language, information and computation, volume 4576 of Lecture Notes in Comput. Sci., pages 285-296. Springer, Berlin, 2007.

[23] Paulo Oliva. An analysis of Gödel's Dialectica interpretation via linear logic. Dialectica, 62(2):269-290, 2008.

[24] Paulo Oliva. Functional interpretations of linear and intuitionistic logic. Inform. and Comput., 208(5):565-577, 2010.

[25] Paulo Oliva. Hybrid functional interpretations of linear and intuitionistic logic. J. Logic Comput., 22(2):305-328, 2012.

[26] Paulo Oliva. Kreisel's modified realizability and recent variants. Kreisel volume, to appear.

[27] Paulo Oliva and Thomas Streicher. On Krivine's realizability interpretation of classical second-order arithmetic. Fundamenta Informaticae, 84(2):207220, September 2008.

[28] Martin Stein. Interpretationen der Heyting-Arithmetik endlicher Typen. Arch. Math. Logik Grundlag, 19:175-189, 1979.

[29] Thomas Streicher and Ulrich Kohlenbach. Shoenfield is Gödel after Krivine. Mathematical Logic Quarterly, 53(2):176-179, April 2007. 